\title{
1 Integrated Multi-omic Framework of the Plant Response to Jasmonic Acid
}

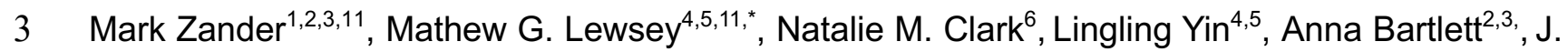

4 Paola Saldierna Guzmán ${ }^{1,7}$, Elizabeth Hann ${ }^{1,8}$, Amber E. Langford ${ }^{1}$, Bruce Jow ${ }^{2,3}$, Aaron Wise ${ }^{9}$,

5 Joseph R. Nery ${ }^{2,3}$, Huaming Chen ${ }^{2}$, Ziv Bar-Joseph ${ }^{9}$, Justin W. Walley ${ }^{6}$, Roberto Solano ${ }^{10}$ and

6 Joseph R. Ecker ${ }^{1,2,3, *}$

$8 \quad{ }^{1}$ Plant Biology Laboratory, Salk Institute for Biological Studies, La Jolla, CA 92037, USA

$9 \quad{ }^{2}$ Genomic Analysis Laboratory, Salk Institute for Biological Studies, La Jolla, CA 92037, USA

$10{ }^{3}$ Howard Hughes Medical Institute, Salk Institute for Biological Studies, La Jolla, CA 92037, USA

$11{ }^{4}$ Centre for AgriBioscience, Department of Animal, Plant and Soil Sciences, School of Life

12 Sciences, La Trobe University, Melbourne, VIC 3086, Australia

$13{ }^{5}$ Australian Research Council Industrial Transformation Research Hub for Medicinal Agriculture,

14 Centre for AgriBioscience, La Trobe University, Bundoora, VIC 3086, Australia

15 'Plant Pathology and Microbiology, lowa State University, Ames, IA 50011, USA

$16{ }^{7}$ Present address: School of Natural Sciences, University of California Merced, Merced, CA

1795343, USA

$18{ }^{8}$ Present address: Department of Chemical and Environmental Engineering, Department of

19 Botany and Plant Sciences, University of California, Riverside, CA 92521, USA

$20{ }^{9}$ Computational Biology Department, School of Computer Science, Carnegie Mellon University,

21 Pittsburgh, PA 15213, USA

$22{ }^{10}$ Department of Plant Molecular Genetics, Centro Nacional de Biotecnología, Consejo Superior

23 de Investigaciones Científicas (CNB-CSIC), 28049 Madrid, Spain

$24 \quad{ }^{11}$ These authors contributed equally

25 *Authors for correspondence: Joseph R. Ecker (ecker@salk.edu), Mathew G. Lewsey 26 (m.lewsey@latrobe.edu.au) 


\section{Abstract}

28 Understanding the systems-level actions of transcriptional responses to hormones provides

29 insight into how the genome is reprogrammed in response to environmental stimuli. Here, we

30 investigate the signaling pathway of the hormone jasmonic acid (JA), which controls a plethora of

31 critically important processes in plants and is orchestrated by the transcription factor MYC2 and

32 its closest relatives in Arabidopsis thaliana. We generated an integrated framework of the

33 response to JA that spans from the activity of master and secondary-regulatory transcription

34 factors, through gene expression outputs and alternative splicing to protein abundance changes,

35 protein phosphorylation and chromatin remodeling. We integrated time series transcriptome

36 analysis with (phospho)proteomic data to reconstruct gene regulatory network models. These

37 enable us to predict previously unknown points of crosstalk from JA to other signaling pathways

38 and to identify new components of the JA regulatory mechanism, which we validated through

39 targeted mutant analysis. These results provide a comprehensive understanding of how a plant

40 hormone remodels cellular functions and plant behavior, the general principles of which provide

41 a framework for analysis of cross-regulation between other hormone and stress signaling

42 pathways. 


\section{Introduction}

54 Plant hormones are structurally unrelated small signaling molecules that play pivotal roles in a

55 wide range of fundamental processes of plants spanning growth, development and responses to 56 environmental stimuli (Vanstraelen and Benkova, 2012). Hormone perception by plants

57 stimulates a cascade of transcriptional reprogramming that ultimately modifies cellular function

58 and plant behavior (Chang et al., 2013, Song et al., 2016, Hickman et al., 2017, Pauwels et al.,

59 2008). This is initiated by one or a family of high-affinity receptors, followed by signal transduction

60 through protein-protein interactions, post-translational modification events and regulation of

61 transcription factor (TF) activity that ultimately drives changes in gene expression (Wang et al.,

62 2015, Song et al., 2016, Chang et al., 2013).

One of the key plant hormones is methyl jasmonate (JA), which regulates crucial processes including fertility, seedling emergence, the response to wounding and the growthdefense balance (Huang et al., 2017). Jasmonates are perceived as jasmonoyl-isoleucine (JAIle) by the co-receptor COI1 (CORONATINE INSENSITIVE1)/JAZ (Jasmonate ZIM domain) complex (Thines et al., 2007, Chini et al., 2007, Fonseca et al., 2009, Sheard et al., 2010). COI1 is an F-box protein and part of a Skp-Cullin-F-box-E3 ubiquitin ligase complex (SCF ${ }^{\mathrm{CO} 11}$ ) (Xie et al., 1998) that targets JAZ proteins for proteasomal degradation upon JA perception. JAZs are transcriptional repressor proteins that inhibit the activity of key TFs of the JA pathway such as the

71 basic helix-loop-helix (bHLH) TF MYC2 and its closest homologues MYC3, MYC4 and MYC5

72 (Fernandez-Calvo et al., 2011, Song et al., 2017, Lorenzo et al., 2004) in the absence of JA. The

73 SCF ${ }^{\mathrm{CO} 11}$-JAZ complex tightly controls the level of free non-repressed MYCs in a JA-dependent

74 manner thereby determining the transcriptional output of the entire JA response (Chini et al.,

75 2007, Thines et al., 2007, Zhang et al., 2015a). The key regulatory step in the JA pathway is the

76 hormone-triggered formation of a complex between the E3 ligase SCF ${ }^{\mathrm{CO} I 1}$ and JAZ repressors

77 that are bound to the master TF MYC2. This results in degradation of JAZ repressors and permits

78 the activity of a master regulatory bHLH transcription factor MYC2, accompanied by MYC3, 
MYC4, MYC5 and numerous other transcription factors, all of which have distinct but overlapping

80 roles in driving JA-responsive gene expression (Song et al., 2017, Schweizer et al., 2013b,

81 Fernandez-Calvo et al., 2011, Lorenzo et al., 2004, Bao et al., 2019). The result is a cascade of

82 JA-induced genome reprogramming to modulate plant behavior such as plant immune responses

83 (Du et al., 2017, Hickman et al., 2017). However, our knowledge of the JA-responsive genome

84 regulatory program and, more broadly, in the plants general response to environmental stimuli is

85 limited currently by assessment of only one or a small number of components.

Here we aim to decipher MYC2/MYC3-driven JA-responsive gene expression using

87 a multi-omics analysis that includes the direct targets of key transcription factors, chromatin

88 modifications, global protein abundance and protein phosphorylation. We discovered that

89 MYC2/MYC3 directly target hundreds of TFs, resulting in a large transcriptional network that

90 facilitates extensive crosstalk with other signaling pathways. This model predicted new

91 components of the JA signaling pathway that we validated by targeted genetic analyses,

92 demonstrating the power of our integrated multi-omic approach to yield fundamental biological

93 insight into plant hormone responses.

\section{Results}

\section{MYC2 and MYC3 binding is associated with a large proportion of JA-responsive genes}

97 To decipher the JA-governed regulatory network with its high degree of dynamic and spatio-

98 temporal interconnectivity with other signaling pathways, we applied a multi-omic network

99 approach that is comprised of five newly generated large-scale datasets (Fig. 1a, b). MYC2 is the

100 master regulatory transcription factor of JA responses and plants with a null mutation causing a

101 clear decrease in JA sensitivity (Lorenzo et al., 2004). Thus, we included the myc2 (jin1-8

102 SALK_061267) mutant into our analyses (Fig. 1b) (Lorenzo et al., 2004). MYC2 is responsible for

103 strong JA-responsive gene activation and acts additively with MYC3 and MYC4 (Lorenzo et al., 
104 2004, Fernandez-Calvo et al., 2011). myc3 and myc4 single mutants behave like wildtype with

105 regards to JA-induced root growth inhibition. However, in combination with the myc2 mutant, 106 myc2 myc3 double mutants exhibit an increased JA hyposensitivity, almost as pronounced as in 107 myc2 myc3 myc4 triple mutants (Fernandez-Calvo et al., 2011). We consequently selected MYC3 108 for an in-depth analysis.

In order to better understand how the master TFs MYC2 and MYC3 control the JA-

110 induced transcriptional cascade, we determined their genome-wide binding sites using chromatin

111 immunoprecipitation sequencing (ChIP-seq). Four biological replicates of JA-treated (2 hours)

112 three-day-old etiolated Arabidopsis seedlings that express a native promoter-driven and epitope

113 (YPet)-tagged version of MYC2 and three biological replicates of MYC3 (Col-0 MYC2::MYC2-

114 Ypet, Col-0 MYC3::MYC3-Ypet) were used (Gimenez-lbanez et al., 2017). The genome-wide

115 distributions of MYC2 and MYC3 binding sites were highly similar (Fig. 1c, d). We identified 6,736

116 MYC2 and 3,982 MYC3 binding sites of high confidence, equating to 6,178 MYC2 and 4,092

117 MYC3 target genes (Fig. 1d and Supplementary Table 1). Of the target genes identified, 3,847

118 were shared, meaning that almost all MYC3 target genes are also bound by MYC2 (Fig. 1d). Their

119 target genes were enriched for JA-related gene ontology terms and for terms related to other

120 hormones (Zhang et al., 2014, Abe et al., 2003) (Supplementary Fig. 1a). Collectively, these data

121 indicate that MYC2 and MYC3 have the potential to regulate $23.2 \%$ of genes in the Arabidopsis

122 genome (27,655 coding genes). However, binding events are not necessarily regulatory (Chang

123 et al., 2013, Song et al., 2016, Fernandez et al., 2003). We determined that 2,522 genes are

124 differently expressed (false discovery rate, FDR <0.05) after two hours of JA treatment using

125 RNA-seq. A third (843 genes) of JA-modulated genes were directly bound by MYC2 or MYC3

126 (Fig. 1d and Supplementary Table 2). This is consistent with the important role of MYC2/3 in JA-

127 responsive gene expression (Lorenzo et al., 2004, Fernandez-Calvo et al., 2011, Schweizer et

128 al., 2013b). The majority of JA-responsive direct MYC2/3 target genes are transcriptionally 
129 upregulated after JA application indicating that MYC2 and MYC3 predominantly act as 130 transcriptional activators (Supplementary Fig. 1b).

132 or MYC3 binding sites, which is concordant with the observation that they shared a large

133 proportion of their binding sites (Fig. 1e, f). This motif was also of similar sequence to a motif

134 bound by MYC2 determined in vitro (Godoy et al., 2011). The majority of MYC2 and MYC3 binding

135 sites contained the G-Box motif (MYC2: 4,240 of 6,736; MYC3: 3,072 of 3,982; Fig. 1e, f and

136 Supplementary Table 3). However, the absence of the motif from a substantial number of MYC2

137 and MYC3 binding sites suggests the transcription factors may bind indirectly to some sites

138 through partner protein(s).

Master TFs directly target the majority of signaling components in their respective

140 pathway, a phenomenon which has already been observed already for the ethylene, abscisic acid

141 and cytokinin signaling pathways (Chang et al., 2013, Song et al., 2016, Xie et al., 2018). This

142 pattern also holds true for the JA signaling pathway. Our MYC2/MYC3 ChIP-seq analyses

143 determined that approximately two thirds of genes encoding for known JA pathway components

144 (112 of 168 genes for MYC2 and 96 of 168 genes for MYC3) are bound by MYC2 and MYC3

145 (Supplementary Fig. 1c, d and Supplementary Table 4). Interestingly, the majority of all known JA

146 genes that were differentially expressed following JA treatment were bound by MYC2 or MYC3

147 whereas fewer non-differentially expressed known JA genes were directly targeted

148 (Supplementary Fig. 1c and Supplementary Table 4). MYCs initiate various feed forward loops

149 that allow a rapid activation of the transcriptional JA response (Du et al., 2017, Liu et al., 2019).

150 Our ChIP-seq approach revealed that besides the autoregulation of MYC2 and MYC3, they also

151 regulate JA biosynthesis either indirectly through binding to the AP2-ERF transcription factor gene

152 ORA47 (Chen et al., 2016a) or directly by targeting the JA biosynthesis genes LOX2 and AOS2

153 (Supplementary Table 4). In addition, MYCs simultaneously target various negative regulators

154 enabling MYCs to efficiently dampen the JA response pattern. Key negative regulators of JA 
155 signaling are the JAZ repressors, a gene family of 13 members in Arabidopsis (Guo et al., 2018,

156 Chung et al., 2010, Cuellar Perez et al., 2014) which can interact with the adaptor protein NINJA

157 to confer TOPLESS-mediated gene repression (Pauwels et al., 2010). Strikingly, all JAZs and

158 also NINJA are directly bound by MYC2 and MYC3 (Supplementary Fig. 1e), with the probable

159 effect of dampening the JA response thereby preventing excessive activation of JA signaling.

160 MYC2 and MYC3 activate the transcriptional JA response through a large transcription

161 factor network

162 To decipher the MYC2 and MYC3-governed transcriptional regulatory network in more detail, we

163 investigated the relationship between MYC2/MYC3-bound TF-encoding genes and their

164 transcriptional responsiveness to JA treatment. We conducted a JA time course experiment (time

165 points $0,0.25,0.5,1,2,4,8,12,24 \mathrm{~h}$ post JA treatment) identifying a total of 7,377 differentially

166 expressed genes at one or more time points within $24 \mathrm{~h}$ of JA treatment (Supplementary Table

167 2). Differentially expressed genes were categorized into clusters with similar expression trends

168 over time to facilitate visualization of complex expression dynamics and enriched functional

169 annotations (Supplementary Fig. 2a and Supplementary Table 5). The largest upregulated cluster

170 was the "JA cluster" which was enriched for gene ontology (GO) terms associated with JA

171 responses (Fig. 2a). In contrast, the "Cell wall cluster" was the largest cluster of downregulated

172 genes and enriched for GO terms associated with cell wall organization, development and

173 differentiation (Fig. 2b). These two main clusters illustrate the defense-growth trade-off that plants

174 are faced when defense pathways are activated (Huot et al., 2014).

175 Up to $63 \%(0.5 \mathrm{~h} \mathrm{JA}$ treatment) of differentially expressed genes at any given time

176 point were directly bound by MYC2 and/or MYC3 (Fig. 2c), highlighting the important role of MYCs

177 in transcriptionally regulating JA responses. Our analysis also determined that 522 of 1,717

178 known or predicted TFs were differentially expressed within $24 \mathrm{~h}$ of JA treatment (Supplementary

179 Fig. 2b). Half of these (268), representing 36 of 58 TF families, were also direct MYC2 or MYC3 
180 targets (Fig. 2d and Supplementary Fig. 2b) indicating that MYC2 and MYC3 cooperatively control

181 a massive TF network. The three most numerous families (ERFs, bHLHs and MYBs) in the

182 Arabidopsis genome had the most JA-responsive MYC2 or MYC3 targeted members which is

183 concordant with their previously annotated roles in JA responses (Fig. 2d) (Chen et al., 2016b).

184 Plant hormone crosstalk is critical for an appropriate cellular response to environmental stimuli

185 and numerous reports describe that MYC2 connects the JA pathway to other major plant hormone

186 pathways (Hou et al., 2010, Lorenzo et al., 2004, Aleman et al., 2016, Zhang et al., 2014, Cui et

187 al., 2018, Pieterse et al., 2009). To investigate this crosstalk function of MYC2 and MYC3 in more

188 detail, we utilized our ChIP-seq data to determine the number of plant hormone TFs that are

189 bound by MYC2 and MYC3. We found that 37 to $59 \%$ of annotated hormone pathway genes are

190 bound by MYC2 and MYC3 and that their expression changes in response to 24 hours of JA

191 treatment (Supplementary Fig. 2c). In addition, we discovered 122 annotated hormone TFs, with

192 representatives from all hormone pathways, that are bound by MYC2 and MYC3 and 118 of these

193 are differentially expressed (Supplementary Fig. 2d and Supplementary Table 1).

194 We next set out to better understand the target genes of the network of TFs downstream

195 of MYC2 and MYC3. To do so we conducted ChIP-seq or DNA affinity purification sequencing

196 (DAP-seq) (O'malley et al., 2016, Bartlett et al., 2017), on a subset of TFs that were direct MYC2/3

197 targets and rapidly upregulated (within $0.5 \mathrm{~h}$ ) by JA treatment (DREB2B, ATAF2, HY5, RVE2,

198 ZAT18; Fig. 2e) or were members of the upregulated "JA cluster" (TCP23; Fig. 2a). We also

199 included TFs with known roles in JA signaling (ERF1, ORA59, NAC3/ANAC055, WRKY51,

200 ZAT10) (Lorenzo et al., 2003, Pre et al., 2008, Bu et al., 2008, Gao et al., 2011, Pauwels and

201 Goossens, 2008). These TFs formed a highly connected network, with all TFs except DREB2B

202 targeting at least two TFs in the network and being themselves targeted by two TFs (Fig. 2e and

203 Supplementary Table 6). Auto-regulation was common, with seven TFs targeting their own loci

204 (Fig. 2e). The target genes of ZAT10, ANAC055 and ATAF2 were most similar to those of MYC2/3

205 (Fig. 2f). Consistent with this, their target genes shared several significantly enriched gene 
ontology terms (adjusted $\mathrm{p}<0.05)$, suggesting related functions in jasmonate signaling

207 (Supplementary Fig. 2d). ORA59 and ERF1, along with DREB2B, formed a distinct group that 208 targeted a related set of genes (Supplementary Fig. 3a). Notably, ERF1 and ORA59 also shared 209 significant enrichment of a separate set of gene ontology terms with one-another, but that were 210 not enriched amongst MYC2/3 targets. This is consistent with the joint role of ERF1 and ORA59

211 in controlling a pathogen defense arm of JA signaling (Pre et al., 2008, Lorenzo et al., 2003). No

212 gene ontology terms were enriched amongst the targets of DREB2B. WRKY51 and RVE2 had 213 relatively few enriched gene ontology terms but shared most of these with one-another. Most of 214 the terms related to anti-insect defense and were a subset of the enriched MYC2/3-ZAT10215 ANAC055-ATAF2 gene ontology terms (Supplementary Fig. 2a). ZAT10 and ANAC055 are 216 known regulators of anti-insect defense and our results suggest WRKY51 and RVE2 may also be 217 involved in this component of jasmonate responses (Schweizer et al., 2013a). Taken together, 218 our analyses determine that MYC2 and MYC3 shape the dynamic spatiotemporal JA response 219 through the activation of a large TF network that includes various potentially coupled feedforward 220 and feedback loops and that allows extensive cross-communication with other signaling 221 pathways.

\section{MYC2 controls JA-induced epigenomic reprogramming}

223 Reprogramming of the epigenome is an integral part of development and environmental stimulus224 induced gene expression (Feng et al., 2010, Xiao et al., 2017). For example, activation of the 225 transcriptional JA response requires the formation of MYC2/MED25-mediated chromatin looping 226 (Wang et al., 2019). To investigate the extent of JA-induced changes in chromatin architecture 227 and the regulatory importance of MYC2 in this response, we conducted ChIP-seq assays to profile 228 the genome wide occupancy of the histone modification H3K4me3 and the histone variant H2A.Z 229 in untreated/JA-treated $(4 \mathrm{~h}) \mathrm{Col}-0$ and myc2 seedlings. Trimethylation of H3K4me3 marks active 230 and poised genes and the histone variant H2A.Z confers gene responsiveness to environmental 231 stimuli (Rothbart and Strahl, 2014, Coleman-Derr and Zilberman, 2012). mRNA expression was 
232 monitored in parallel using RNA-seq. JA treatment led to a reprogrammed chromatin landscape

233 with several thousand differentially enriched H3K4me3 and H2A.Z domains (Supplementary Fig.

234 4a, b, c and Supplementary Table 7). We identified 826 differentially expressed genes (675

235 induced, 151 repressed; Col-0 control v. JA-treated) in that experiment and, as expected, the JA-

236 induced genes had a stronger promoter enrichment of MYC2 than the JA-repressed genes (Fig.

237 3a and Supplementary Table 2). The JA-induced genes had an increase of H3K4me3, whereas

238 JA-repressed genes had no dynamic change in the level of H3K4me3 (Fig. 3b, d). Strikingly, myc2

239 mutants only display a compromised increase of H3K4me3 after JA treatment suggesting that the

240 JA-induced trimethylation of H3K4me3 strongly depends on prior MYC2 binding (Fig. 3b, c, d and

241 Supplementary Fig. 4a). This scenario is illustrated by two JA-induced genes, JAZ2 and GRX480,

242 which are directly targeted by MYC2. Their expression depends on MYC2 and their JA-induced

243 increase of gene body-localized H3K4me3 partially depends on MYC2 (Fig. 3d and

244 Supplementary Fig. 4d). In contrast, JA-induced changes in H2A.Z occupancy are not affected in

245 myc2 mutants (Supplementary Fig. 4a) suggesting that JA-induced H2A.Z dynamics are either

246 independent of MYC2 or other MYCs such as MYC3, MYC4 and MYC5 are functionally redundant

247 in regulating H2A.Z dynamics.

248 Extensive remodelling of the (phospho)proteome occurs following a JA stimulus and may

249 drive alternative splicing

250 We next explored how JA remodels the proteome and phosphoproteome of etiolated seedlings.

251 Hormone signal transduction typically modifies phosphorylation of downstream proteins,

252 changing their activity independent of transcript abundance (Wang et al., 2015). Transcript

253 abundances are also frequently weakly correlated with protein abundances (Walley et al., 2016,

254 Baerenfaller et al., 2008). Consequently, proteomic and phosphoproteomic analyses yield

255 additional insight into gene regulatory networks. We determined that the loss of MYC2 caused

256 substantial changes to the JA-responsive proteome and phosphoproteome; 1,432 proteins and 
257939 phosphopeptides (corresponding to 567 genes) were significantly differentially abundant in 258 Col-0 seedlings relative to myc2 seedlings after $2 \mathrm{~h}$ JA treatment $(q<0.01 ;$ Fig. $4 a$ and 259 Supplementary Table 8, 9). Col-0 seedlings responded to JA (161 proteins, 443 phosphopeptides, 260 Col-0 JA v. Col-0 air) and the response was smaller without functional MYC2 (79 proteins, 93 261 phosphopeptides, myc2 JA v. myc2 air). Some overlap existed between proteins or 262 phosphopeptides and transcripts responsive to JA treatment (Fig. 4b). Both transcripts and 263 proteins encoded by 28 genes were differentially expressed in JA-treated Col-0 seedlings relative 264 to air controls. A further 33 differentially expressed proteins in JA-treated Col-0 seedlings had no 265 corresponding differentially expressed transcript but were encoded by genes that are targeted by 266 MYC2 and MYC3. Differentially abundant phosphopeptides were detected that corresponded with 26715 differentially expressed transcripts. Transcript and protein abundances also correlated poorly 268 in JA-treated Col-0 seedlings (Fig. 4c), in agreement with prior studies (Walley et al., 2016, 269 Baerenfaller et al., 2008). The protein of only one known JA pathway component was differentially 270 abundant in JA-treated Col-0 seedlings relative to controls, and none were differentially 271 phosphorylated. In sum, these data indicate that the JA-responsive proteome and 272 phosphoproteome are poorly annotated and are not well represented by transcriptome studies 273 (Fig. 4c).

Alternative splicing can occur rapidly in response to environmental stimuli, 275 contributing to transcriptome reprogramming and potentially fine-tuning physiological responses 276 (Hartmann et al., 2016, Calixto et al., 2018). It is central to JA-mediated regulation of transcription, 277 with an alternative isoform of the repressor JAZ10 creating a negative feedback loop that 278 desensitizes cells to a JA stimulus (Moreno et al., 2013, Zhang et al., 2017, Chung et al., 2010). 279 However, the extent of alternative splicing in JA signaling beyond the JAZ repressors is poorly 280 characterized. We observed that phosphorylation of proteins involved in RNA recognition and 281 nucleotide binding was disrupted in JA-treated myc2 mutants compared with Col-0 seedlings. The 282 spliceosome was the only pathway significantly enriched amongst these differentially 
283 phosphorylated proteins ( $p<0.05,18$ genes matched) suggesting that MYC2 may influence JA-

284 responsive alternative splicing. We examined isoform switching events across our JA

285 transcriptome time-series, where the most abundant of two isoforms from a single gene changes,

286 to determine the extent of JA-responsive alternative splicing (Fig. 4d, e, Supplementary Table

287 10). There were 151 switch events, corresponding to 137 isoform pairs from 120 genes, within 24

288 h of JA treatment. These were identified from 30,547 total individual transcripts detected (average

289 TPM>1; Supplementary Table 11). Two of the genes exhibiting isoform switches had prior JA

290 annotations (RVE8/AT3G09600, SEN1/AT4G35770) and others were annotated to a variety of

291 processes (including auxin, ABA, light signaling, disease response, amongst many others), but

292 there was no significant enrichment of any gene ontology terms or pathways. This indicates that

293 MYC2 influences alternative splicing that diversifies the transcriptome in response to a JA

294 stimulus.

295 Multi-omic modelling provides a comprehensive understanding of the JA response 296 genome regulatory program

297 We wanted next to characterize the broader JA-response genome regulatory program so that we 298 could increase our understanding of the roles of known JA TFs within this and identify new 299 potential regulatory interactions. To do so we generated a gene regulatory network model 300 encompassing the (phospho)proteomic and time-series transcriptomic data (Supplementary Fig.

$3015 a$ and Supplementary Table 12). Many known JA signaling components were present in the 100 302 most important predicted components of the model (MYC2, ERF1, JAZ1, JAZ2, JAZ5, JAZ10, 303 ATAF2 and others; within top 100 of 4366 components by normalized motif score; Supplementary 304 Table 12). MYC2 was predicted to regulate a subnetwork of 26 components, 23 of which were 305 validated as directly bound by MYC2 in ChIP-seq assays (88.5\%, Supplementary Fig. 6a and 306 Supplementary Table 1, 12). We further validated the network by comparing the ChIP/DAP-seq 307 data previously collected for the remaining 12 JA TFs to their targets in the gene regulatory 
network (Fig. 2, Supplementary Fig. 6b and Supplementary Table 13). The gene regulatory network identified all of these TFs as components of the JA response, except MYC3

310 (Supplementary Table 12). It is likely that MYC3 was not part of the network due to it being only 311 modestly differentially expressed following JA treatment and not being detected in the 312 (phospho)proteome analyses (Supplementary Tables 2, 8, 9). The wider validation of targets was 313 less strong than for MYC2, ranging from $0 \%$ to $33.3 \%$. This could reflect the possibility that 314 interactions predicted by the gene regulatory network may not identify all intermediate 315 components. Lastly, we examined known genetic interactions. The MYC2 subnetwork included 316 activation of JAZ10 within $0.5 \mathrm{~h}$ of a JA stimulus, with JAZ10 reciprocally repressing MYC2

317 (Supplementary Fig. 6a, b). This is consistent with the known role of JAZ10 in establishing 318 negative feedback that attenuates JA signaling (Moreno et al., 2013). MYC2 was also predicted 319 to activate AIB (JAM1/bHLH017/AT2G46510) (Supplementary Fig. 6a, b), establishing a negative feedback loop in which AIB negatively regulates MYC2. This is consistent with prior studies, which established AIB is dependent upon and antagonistic to MYC2, thereby repressing JA signaling

322 (Nakata et al., 2013, Sasaki-Sekimoto et al., 2013, Fonseca et al., 2014). Confirmation by both 323 genetic data from the literature and our DAP/ChIP-seq experiments indicates that our gene 324 regulatory network modelling approach is a useful tool to identify new regulatory interactions 325 within JA signaling and to better understand known regulatory interactions.

Crosstalk between hormone response pathways permits fine-tuning of plant growth

327 and development in response to diverse environmental signals (Karasov et al., 2017). We 328 examined the potential points at which MYC2 may interface directly with other hormone signaling 329 pathways, since MYC2 is the master regulator of JA responses and one of the first TFs activated 330 by JA. The MYC2 subnetwork identified a potential route for JA signaling to cross-regulate auxin 331 hormone signaling. MYC2 activated ARF18 and ARF18 reciprocally activated MYC2

332 (Supplementary Fig. 6a and Supplementary Table 12). It also indicated that MYC2 may promote 333 ethylene signaling by activating MAP kinase kinase 9 (MKK9) (Supplementary Fig. 6a). Prior 
334 genetic studies determined that MKK9 induces ethylene production, but had not examined a 335 possible link with JA signaling (Xu et al., 2008). Positive crosstalk is known to exist between JA 336 and auxin signaling though the mechanism is not clearly determined (An et al., 2010, Hentrich et 337 al., 2013). RGL3, a regulator of gibberellic acid (GA) signaling previously associated with JA-GA 338 crosstalk, was also present within the MYC2 subnetwork (Supplementary Fig. 6a), predicted to 339 inhibit MYC2 but not to be reciprocally regulated by MYC2 (Wild et al., 2012). These three 340 interactions are potential points at which crosstalk can occur rapidly during a JA response with 341 auxin, gibberellin and ethylene.

342 We next examined the broader gene regulatory network to identify additional 343 predicted points of crosstalk between JA and other signaling pathways. The model predicted that 344 STZ/ZAT10 is a key early hub through which JA signaling is prioritized over several other hormone 345 and stress response pathways (Fig. 5a and Supplementary Table 12). STZ/ZAT10 is known to be 346 a transcriptional repressor from genetic studies (Mittler et al., 2006) and, consistent with this, our 347 model predicted that it inhibited the majority of genes it regulates (25 of 34 genes). WRKY40, 348 WRKY70, DDF and ERF6 were all predicted to be inhibited by STZ/ZAT10 within $0.25 \mathrm{~h}$ of a JA 349 stimulus and GRX480 within $1 \mathrm{~h}$. Direct binding of STZ/ZAT10 to ERF6 was detected in ChIP-seq 350 assays (Supplementary Table 6). WRKY40 and WRKY70 both are both early brassinosteroid 351 response components that repress defense responses (Lozano-Duran et al., 2013). WRKY70 352 also fine-tunes the crosstalk between the salicylic acid and JA pathways (Li et al., 2006). DDF1 353 promotes resistance to drought, cold, heat and salinity stress by reducing endogenous gibberellin 354 abundance (Kang et al., 2011, Magome et al., 2008). ERF6 similarly promotes drought resistance 355 by reducing gibberellin abundance (Dubois et al., 2015). GRX480 regulates the negative crosstalk 356 between salicylic acid and both JA/ethylene signaling through the direct interactions with TGA 357 transcription factors (Zander et al., 2012, Ndamukong et al., 2007). The model also predicts that 358 ERF6, WRKY70 and DDF1 exert negative feedback on STZ/ZAT10 by activating JAZ8 within 0.25 $359 \mathrm{~h}$ of the JA stimulus (Fig. 5a and Supplementary Table 12). JAZ8 is a known repressor of JA 
360 signaling and is predicted to repress STZ/ZAT10 (Shyu et al., 2012). In sum the gene regulatory 361 network predicts that STZ/ZAT10 is an important hub for JA signaling to be prioritized over other 362 hormone and stress response pathways (Fig. 5a).

363 Phenotypic screening guided by large-scale data identifies new JA signaling components 364 and validates the JA gene regulatory network

365 We next utilized our regulatory network and large-scale datasets to identify novel regulators of 366 the JA pathway using the JA root growth inhibition assay as our experimental readout. First, we 367 focused on ABO3 (ABA overly sensitive 3), which is directly targeted by MYC2 and MYC3 (Fig.

$3682 \mathrm{~d}$ and Supplementary Table 1) and whose subnetwork is comprised of 26 predicted regulated 369 genes, the majority of which is positively regulated (22 of 26 genes) (Fig. 5b). ABO3 encodes the 370 Arabidopsis WRKY transcription factor gene WRKY63, which is involved in stress gene 371 expression and drought tolerance (Ren et al., 2010, Van Aken et al., 2013). To investigate the 372 importance of the $\mathrm{ABO} 3$ subnetwork in JA signaling, we tested abo3 T-DNA mutant seedlings 373 (SALK_075986C) in a JA-induced root growth inhibition assay. We found that abo3 mutants show 374 a weak JA hypo-sensitive root growth inhibition phenotype (Fig. 5c-e) indicating that $\mathrm{ABO} 3$ is 375 positive regulator of JA signaling and that our network approach is able to identify new pathway 376 components.

377 Next, we expanded our phenotyping analysis to T-DNA lines of genes that display the 378 strongest binding of MYC2 and MYC3 in their promoters (Supplementary Table 1, 13). The 379 rationale behind this approach is that master TFs target the majority of key signaling components 380 in their regulated respective pathways and that these are often the most strongly bound targets 381 (Chang et al., 2013, Song et al., 2016, Xie et al., 2018). Of 99 genes tested (194 T-DNA lines in 382 total, Supplementary Table 14), we discovered six genes, when mutated, display mild JA root 383 growth phenotypes (Supplementary Fig. 7a and Supplementary Table 14). Mild phenotypes as 384 well as their low frequency were not surprising since gene redundancy is very common in the 
Arabidopsis genome and even the mutation of the master TF MYC2 only causes a mild JAhyposensitive root growth phenotype (Fig. 5c-e) (Lorenzo et al., 2004, 2000). Among these genes was the cytochrome P450 enzyme CYP708A2 gene from which both tested T-DNA mutant alleles exhibit a JA hypersensitive root phenotype (Fig. 5f-h). Interestingly, our network analysis also discovered CYP708A2 as a regulatory hub (Supplementary Fig. 5a, 7b). CYP708A2 is involved in the triterpene synthesis which is known to be stimulated by methyl jasmonate (Field and Osbourn, 2008, Mangas et al., 2006); future studies are however needed to further decipher the role of CYP708A2 in JA signaling. Another interesting uncharacterized gene that we discovered caused a JA phenotype is a Sec14p-like phosphatidylinositol transfer family protein (AT5G47730)

394 (Supplementary Fig. 7a and Supplementary Table 14). Phosphatidylinositol transfer proteins 395 (PITPs) are crucial for the phosphatidylinositol homeostasis in plants (Huang et al., 2016) and 396 inositol polyphosphates have been implicated in COI1-mediated JA perception (Mosblech et al., 397 2011). Taken together, these data show that our multi-omic approach goes beyond network description ultimately enabling the identification of novel JA pathway regulators.

\section{Discussion}

An important unanswered question in plant biology is how multiple signaling pathways interact to

401 coordinate control of growth and development. In this study we have comprehensively 402 characterized the cellular response to the plant hormone JA and generated a network-level 403 understanding of the MYC2/MYC3-regulated JA signaling pathway. We used this to identify 404 several new points at which JA signaling may have cross-regulation with other hormone and 405 stress response pathways in order to prioritize itself. The results increase knowledge of how JA 406 functions in the etiolated seedling, a less well characterized model for JA responses. Moreover, 407 the general principles described here provide a framework for analysis of cross-regulation 408 between hormone and stress signaling pathways. We provide our data in a web-based genome 
and network

browsers

to

encourage

deeper

exploration

410 (http://signal.salk.edu/interactome/JA.php, http://neomorph.salk.edu/MYC2).

The major insight provided by our study is that multiple points of crosstalk are likely to

412 exist between JA signaling and other pathways. This was evident from the interactions within the

413 genome regulatory network model and supported by our observation that many (37 to 59\%) genes

414 from other hormone signaling pathways are bound by MYC2/3 and JA-regulated. The WRKY

415 family $\mathrm{TF} \mathrm{ABO} 3$ was identified as a candidate JA response regulator and genetic analyses

416 determined a mutant of the gene was JA hyposensitive. $\mathrm{ABO} 3$ is also a regulator of $\mathrm{ABA}$

417 responses (Ren et al., 2010) suggesting that $\mathrm{ABO} 3$ functions in the cross-communication

418 between the JA and ABA pathway. The repressive zinc-finger family TF STZ/ZAT10, working with

419 JAZ8, emerged as a potentially important point of contact with salt and drought stress, as well as

420 the salicylic acid, brassinosteroid and gibberellin hormone signaling pathways. Combined these

421 results illustrate the importance of transcriptional cross-regulation during a JA response in

422 modulating the correct cellular output for the stimuli a plant perceives.

Our multi-omic analysis determined that the master TF MYC2 and its relative MYC3

424 directly target thousands of JA responsive genes including hundreds of JA responsive TFs,

425 thereby enabling a robust cascade of transcriptional reprogramming. Secondary TFs downstream

426 of MYC2/3 directly targeted overlapping but distinct cohorts of genes, indicating they have distinct

427 roles within the JA response. This illustrates the complexity of hormone-response genome

428 regulatory programs; we have assayed only a fraction of the JA-responsive TFs and find that any

429 individual JA-responsive gene may be bound by multiple TFs. How the final quantitative output of

430 any individual gene is determined by combinatorial binding of TFs remains a major challenge to

431 address. We further demonstrated the importance of MYC2/3 target genes in JA responses by

432 analyzing JA root growth phenotypes in mutants of 99 genes strongly targeted by MYC2/3.

433 Mutations in seven genes caused clear disruption of JA responses, both hyper and hypo- 
434 sensitivity. It is probable that genetic redundancy accounts for a proportion of the mutants not

435 causing phenotype changes.

436 Another layer of regulatory complexity within the JA signaling pathway, and within

437 signaling pathways in general, is the presence of multiple feedforward and feedback loops that

438 are activated simultaneously. The interactions between these subnetworks through their kinetics

439 and the strength of their regulatory impact on the broader network is not well understood. For

440 example, we discovered that MYC2 and MYC3 stimulate JA biosynthesis but also target the entire

441 JAZ repressor family from which the majority of members is also transcriptionally activated.

442 Uncoupling these subnetworks would be an effective way to determine how they interact to drive

443 very robust activation of the JA pathway. The combination of our multi-omic framework approach

444 coupled with powerful genetic approaches such as the generation of the jaz decuple mutant (Guo

445 et al., 2018) should significantly contribute to a better understanding of JA response pathways

447 Acknowledgements: We thank several postdocs, undergrads and technicians who contributed

448 technical assistance to the project; Mingtang Xie, Liang Song, Raul Carlos Serrano, Candice Sy,

449 Lourdes Tames, Julie Park, Omar Romero, Raymond Luong, Waina Ho, Yusuke Koga, Sasha

450 Hazelton, Mark Urich, Tsegaye Dabi. We thank Shao-shan Carol Huang for computational

451 assistance and James Moresco and Jolene Diedrich for proteomics support.

452 Funding: M.Z. was supported by a Deutsche Forschungsgemeinschaft (DFG) research 453 fellowship (Za-730/1-1) and also by the Salk Pioneer Postdoctoral Endowment Fund. M.G.L. was 454 supported by an EU Marie Curie FP7 International Outgoing Fellowship (252475). In addition, this 455 work was supported by the Mass Spectrometry Core of the Salk Institute with funding from NIH$456 \mathrm{NCl}$ CCSG (P30 014195) and the Helmsley Center for Genomic Medicine. This work was 457 supported by grants from the National Science Foundation (NSF) (MCB-1818160 and IOS4581759023 to J.W.W, MCB-1024999 to J.R.E), the National Institutes of Health (R01GM120316), 
459 the Division of Chemical Sciences, Geosciences, and Biosciences, Office of Basic Energy

460 Sciences of the U.S. Department of Energy (DE-FG02-04ER15517) and the Gordon and Betty

461 Moore Foundation (GBMF3034). Research in R.S. lab was supported by grant BIO2016-77216-

$462 \mathrm{R}$ (MINECO/FEDER) from the Ministry of Economy, Industry and Competitiveness. J.W.W. is

463 supported as a Faculty Scholar of the ISU Plant Sciences Institute. J.R.E. is an Investigator of the

464 Howard Hughes Medical Institute.

465 Contributions: M.Z., M.G.L., R.S. and J.R.E. designed the research. M.Z., M.G.L., A.E.L. and

466 B.J. performed the phenotype screening. M.Z., M.G.L. and J.P.S.G. carried out the RNA-seq and

467 ChIP-seq experiments. M.G.L., E.H. and J.P.S.G. performed the cloning and generation of 468 transgenic constructs. M.G.L., J.R.N., H.C, M.Z. and L.Y. analyzed the sequencing data and 469 performed bioinformatics analyses. A.B. carried out DAP-seq experiments. N.M.C. and J.W.W. 470 analyzed the proteome and phosphoproteome data. N.M.C., J.W.W., A.W., S.J. and Z. B-J. 471 performed regulatory network analyses. M.Z., M.G.L and J.R.E. prepared the figures and wrote 472 the manuscript.

473 Competing interests: Authors declare no competing interests.

474 Data and material availability: All described lines can be requested from the corresponding 475 author. Sequence data can be downloaded from GEO (GSE133408). Proteomics data are 476 deposited at Proteome Exchange under the accession ID PXD013592. Visualized data can be 477 found under http://neomorph.salk.edu/MYC2 and http://signal.salk.edu/interactome/JA.php. 


\section{Material \& Methods}

\section{Plant material and growth conditions}

484 The myc2 mutant in this study is jin1-8 (SALK_061267) (Lorenzo et al., 2004) and was obtained 485 from the Arabidopsis Biological Resource Center (ABRC). Col-0 MYC2::MYC2-Ypet and Col-0 486 MYC3::MYC3-Ypet, generated by recombineering, have been described previously (Zhou et al., 487 2011). For the generation of all large scale datasets, three-day-old etiolated seedlings were used 488 (Col-0, myc2, MYC2::MYC2-Ypet, MYC3::MYC3-Ypet). Gaseous MeJA treatment for the 489 respective times was performed as previously described (Schweizer et al., 2013b). For the JA490 induced root growth inhibition assay, surface-sterilized Col-0, myc2 and T-DNA mutant seeds 491 (Supplementary Table 13) were grown on agar plates containing LS medium supplemented with 492 or without $50 \mu \mathrm{M}$ MeJA (392707, Millipore Sigma) for 9 days. Plates were scanned afterwards 493 and root length was measured using ImageJ.

\section{ChIP-seq}

495 Three-day-old etiolated Col-0 MYC2::MYC2-Ypet, Col-0 MYC3::MYC3-Ypet, Col-0 and myc2 seedlings were used for ChIP-seq experiments. ChIP assays were performed as previously 497 described (Kaufmann et al., 2010). ChIP-seq assays were conducted with antibodies against 498 H2A.Z (39647, Active Motif), H3K4me3 (04-745, Millipore Sigma) and GFP (11814460001, 499 Millipore Sigma or goat anti-GFP supplied by David Dreschel, Max Planck Institute of Molecular 500 Cell Biology and Genetics). As a negative control, mouse or goat IgG (015-000-003 or 005-000$501 \quad 003$, Jackson ImmunoResearch) was used. The respective antibodies or IgG were coupled for 4-

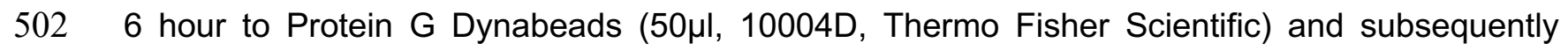
503 incubated overnight with equal amounts of sonicated chromatin. Beads were washed twice with 504 high salt buffer (50 mM Tris $\mathrm{HCl} \mathrm{pH} \mathrm{7.4,} 150$ mM NaCl, 2 mM EDTA, 0.5\% Triton X-100) low salt 505 (50 mM Tris $\mathrm{HCl} \mathrm{pH} \mathrm{7.4,} 500 \mathrm{mM} \mathrm{NaCl}, 2 \mathrm{mM}$ EDTA, 0.5\% Triton X-100) and wash buffer (50 mM 506 Tris $\mathrm{HCl} \mathrm{pH} \mathrm{7.4,} 50 \mathrm{mM} \mathrm{NaCl}, 2 \mathrm{mM}$ EDTA) before samples were de-crosslinked, digested with 
507 proteinase $\mathrm{K}$ and DNA was precipitated. Sequencing libraries were generated following the

508 manufacturer's instructions (Illumina). Libraries were sequenced on the Illumina HiSeq 2500 and

509 HiSeq 4000 Sequencing system and sequencing reads were aligned to the TAIR10 genome

510 assembly using Bowtie2 (Langmead, 2010).

\section{DAP-seq}

512 DAP-seq assays were carried as previously described (O'malley et al., 2016) using recombinantly

513 expressed ERF1 (AT3G23240, ERF1B, AtERF092), ORA59 (AT1G06160), ATAF1

514 (AT1G01720), DREB2B (AT3G11020), ZAT18 (AT3G53600), RVE2 (AT5G37260), WRKY51

515 (AT5G64810), HY5 (AT5G11260) and TCP23 (AT1G35560).

\section{RNA-seq}

517 Three-day-old etiolated seedlings were used for expression analyses. Total RNA was extracted

518 with the RNeasy Plant Mini Kit (74903, Qiagen). cDNA library preparation and subsequent single

519 read sequencing was carried as previously described (Song et al., 2016).

\section{RNA-seq analyses}

521 Sequencing reads were quality trimmed using TrimGalore 0.4 .5

522 https://github.com/FelixKrueger/TrimGalore) then aligned to the TAIR10 genome assembly using

523 TopHat 2.1.1 (Kim et al., 2013). Reads within gene models were counted using HTSeq (Anders

524 et al., 2015). Differentially expressed genes in time series RNA-seq were identified using EdgeR

$525 \quad 3.6 .2$ with a likelihood ratio test (functions glmFit and glmLRT), batch correction Benjamini \&

526 Hochberg correction for multiple tests (Robinson et al., 2010). Differentially expressed genes in

527 the Col-0 versus myc2 mutant RNA-seq were determined using EdgeR 3.18 .1 and quasi-

528 likelihood F-tests (function glmQLFit) (Lun et al., 2016). Temporal co-regulation of transcripts was

529 determined using the Short Time-Series Expression Miner (Ernst and Bar-Joseph, 2006). Known

530 A. thaliana TFs were identified by reference to PlantTFDB 4.0 (Jin et al., 2017). 


\section{ChIP-seq and DAP-seq analyses}

533 ChIP-seq and DAP-seq sequence reads were mapped to the TAIR10 reference genome using

534 Bowtie 2 v.2-2.0.5 with default parameters (Langmead and Salzberg, 2012). For TF ChIP-seq,

535 enriched binding sites were identified using MACS2 v.2.1 (options -p 99e-2 --nomodel -shiftsize

536 --down-sample --call-summits) against sequence reads from whole IgG control samples (Zhang

537 et al., 2008). The shift size was calculated using PhantomPeakQualTools v.2.0 (Kharchenko et

538 al., 2008). Subsequent analyses used summits only. Summit lists were filtered with a lower cut-

539 off of $-\log 10(25)$ and remaining summits expanded from single nucleotides to 150 nt. Only

540 summits with at least $10 \%$ nt overlap between at least two biological replicates were retained.

541 These overlapping summits were merged between replicates using BEDtools v.2.17.0 to give the

542 final set of high-stringency summits, which were then annotated using ChIPpeakAnno v.2.2.0 to

543 any gene within $500 \mathrm{nt}$ of the center of the summit or, alternatively, the nearest neighbor if there

544 was no gene within 500 nt (Quinlan and Hall, 2010, Zhu et al., 2010). Venn diagrams were drawn

545 using Venny and Intervene (http://bioinfogp.cnb.csic.es/tools/venny/) (Khan and Mathelier, 2017).

546 Top-ranked MYC2/3 binding sites were identified by applying IDR to the summits from the two

547 biological replicates that had the greatest number of summits above the MACS2 lower cut-off of

548 -log10(25). TF binding motifs were determined using the MEME-ChIP webserver with default

549 parameters on the sequences of the high-stringency summits (Machanick and Bailey, 2011). The

550 Genome wide Event finding and Motif discovery (GEM) tool (Guo et al., 2012) was used to identify

551 the target summits in DAP-seq data. Significant enrichments of histone modifications and histone

552 variants were identified with the SICER software (Zang et al., 2009) using the TAIR10 genome

553 assembly. The Intersect tool from BEDtools (Quinlan and Hall, 2010) was used to identify the

554 genes in the ChIP-seq datasets that are most proximal to the discovered binding sites. For both

555 ChIP-seq and DAP-seq gene ontology enrichment was assessed using clusterProfiler (Yu et al., 556 2012). 


\section{Mass spectrometry analysis}

559 Ground untreated/JA-treated Col-0 and myc2 seedlings tissue was ground and lysed in

560 YeastBuster (71186, Millipore Sigma). Proteins (100 $\mu \mathrm{g}$ per sample) were precipitated using

561 methanol- chloroform. Dried pellets were dissolved in $8 \mathrm{M}$ urea, $100 \mathrm{mM}$ triethylammonium

562 bicarbonate (TEAB), reduced with $5 \mathrm{mM}$ tris (2-carboxyethyl) phosphine hydrochloride (TCEP),

563 and alkylated with $50 \mathrm{mM}$ chloroacetamide. Proteins were then trypsin digested overnight at 37

$564{ }^{\circ} \mathrm{C}$. The digested peptides were labeled with TMT10plex ${ }^{\mathrm{TM}}$ Isobaric Label Reagent Set (90309,

565 Thermo Fisher Scientific, lot TE264412) and combined. One hundred micrograms (the pre-

566 enriched sample) was fractionated by basic reverse phase (84868, Thermo Fisher Scientific).

567 Phospho-peptides were enriched from the remaining sample (900 $\mu \mathrm{g})$ using High-Select Fe-NTA

568 Phospho-peptide Enrichment Kit (A32992, Thermo Fisher Scientific). The TMT labeled samples

569 were analyzed on a Fusion Lumos mass spectrometer (Thermo Fisher Scientific). Samples were

570 injected directly onto a $25 \mathrm{~cm}, 100 \mu \mathrm{m}$ ID column packed with BEH $1.7 \mu \mathrm{m}$ C18 resin (186002350,

571 Waters) and subsequently separated at a flow rate of $300 \mathrm{~nL} / \mathrm{min}$ on a $\mathrm{nLC} 1200$ (LC140, Thermo

572 Fisher Scientific). Buffer A and B were $0.1 \%$ formic acid in water and $90 \%$ acetonitrile,

573 respectively. A gradient of $1-20 \%$ B over $180 \mathrm{~min}$, an increase to $40 \%$ B over 30 min, an increase

574 to $100 \%$ B over another 20 min and held at $90 \%$ B for a final 10 min of washing was used for 240

575 min total run time. Column was re-equilibrated with $20 \mu \mathrm{L}$ of buffer A prior to the injection of

576 sample. Peptides were eluted directly from the tip of the column and Nano sprayed directly into

577 the mass spectrometer by application of $2.8 \mathrm{kV}$ voltage at the back of the column. The Lumos

578 was operated in a data dependent mode. Full MS1 scans were collected in the Orbitrap at 120000

579 resolution. The cycle time was set to $3 \mathrm{~s}$, and within this $3 \mathrm{~s}$ the most abundant ions per scan

580 were selected for CID MS/MS in the ion trap. MS3 analysis with multinotch isolation (SPS3) was

581 utilized for detection of TMT reporter ions at 60000 resolution. Monoisotopic precursor selection

582 was enabled and dynamic exclusion was used with exclusion duration of $10 \mathrm{~s}$. 
583 The raw data were analyzed using MaxQuant version 1.6.3.3 (Tyanova et al., 2016). Spectra

584 were searched, using the Andromeda search engine (Cox et al., 2011) against the Tair10 585 proteome file entitled "TAIR10_pep_20101214" that was downloaded from the TAIR website 586 (https://www.arabidopsis.org/download/indexauto.jsp?dir=\%2Fdownload_files\%2FProteins\%2F

587 TAIR10_protein_lists) and was complemented with reverse decoy sequences and common 588 contaminants by MaxQuant. Carbamidomethyl cysteine was set as a fixed modification while 589 methionine oxidation and protein N-terminal acetylation were set as variable modifications. For 590 the phoshoproteome "Phosho STY" was also set as a variable modification. The sample type was 591 set to "Reporter lon MS3" with "10plex TMT selected for both lysine and N-termini". Digestion 592 parameters were set to "specific" and "Trypsin/P;LysC". Up to two missed cleavages were 593 allowed. A false discovery rate, calculated in MaxQuant using a target-decoy strategy (Elias and 594 Gygi, 2010) less than 0.01 at both the peptide spectral match and protein identification level was 595 required. The 'second peptide' option identify co-fragmented peptides was not used. Differentially expressed proteins and phospho-sites were identified using PoissonSeq (Li et al, 2012) with a q-

597 value cutoff of 0.1 . Sample loading normalization was performed before differential expression 598 analysis.

599 Transcript quantification and identification of isoform switches

600 Quantification of transcripts was performed using Salmon v0.8.1 in conjunction with the AtRTD2601 QUASI transcript reference (Patro et al., 2017, Zhang et al., 2015b) The quasi mapping-based 602 index was built using an auxiliary k-mer hash over k-mers of length $31(k=31)$. For quantification, 603 all parameters of Salmon were kept at default, except that the option to correct for the fragment604 level GC biases (“-gcBias”) was turned on.

605 The TSIS R package, which is designed for detecting alternatively spliced isoform switch 606 events in time-series transcriptome data, was used to perform the isoform switch analysis (Guo 607 et al., 2017) Only transcripts whose average transcript per million (TPM) across all time points 608 was $>1$ were included in the TSIS analysis. The mean expression approach was used to search 
609 interaction points. Significant switch events were identified using the following filtering

610 parameters: (1) probability cutoff $>0.5$; (2) differences cutoff $>1$; (3) p-value cutoff $<0.05$; (4) $\min$

\section{Gene regulatory network (GRN) inference}

612 All GRNs were constructed using the Regression Tree Pipeline for Spatial, Temporal, and

613 Replicate data (RTP-STAR) (Shibata et al., 2018) Clark et al., 2018). Prior to GRN inference,

614 genes were clustered based on transcriptome, proteome, or phosphoproteome data using

615 Dynamic Time Warping (DTW) (and the dtwclust package in R (Giorgino 2009). These clusters

616 were then used in the RTP-STAR pipeline. For the transcriptome networks, one network was

617 inferred for genes differentially expressed at each time point (8 networks total), and then the

618 networks were combined in a union. For each network, the biological replicates for that individual

619 time point and the $0 \mathrm{~h}$ (control) time point were used to infer the network. The sign

620 (activation/repression) of each edge was inferred using all of the time points in the time course.

621 For the proteome and phosphoproteome networks, one network was inferred for genes

622 differentially expressed in any of the comparisons. The biological replicates for all of the (phospho)

623 proteome samples were used to infer the network. The sign of each edge was not inferred as the

624 (phospho) proteome data only consisted of one time point.

625 After the transcriptome, proteome, and phosphoproteome networks were combined in a union, a

626 Network Motif Score (NMS; Clark et al., 2018) was calculated to determine the importance of

627 each gene. Feedback loop, feed-forward loop, bi-fan, and diamond motifs were used in this score

628 as they have been previously shown to contain genes important for biological processes (Alon,

629 2007, Milo et al., 2002, Ingram et al., 2006). All motifs were significantly enriched in the combined

630 network compared to a randomly generated network of the same size. The number of times each

631 gene appeared in each motif was counted, the counts were normalized to a scale of 0 to 1 , and

632 the counts were summed to calculate the NMS. The higher the NMS, the more functionally

633 important the gene is. All code for RTP-STAR is available at https://github.com/nmclark2/RTP- 
634 STAR. The parameters used for all networks in this paper are provided in Supplementary Table

63515.

636 Figure legends

637 Figure 1. Design of our study and key datasets utilized.

638 a, b, Overview of profiled regulatory layers (a) and detailed description of collected samples (b).

639 c, AnnoJ genome browser screenshot visualizes the binding of MYC2 and MYC3 to the three

640 example genes (IAR3/UR3, ACT1, JAZ9/TIFY7). MYC2/3 binding was determined with ChIP-seq

641 using JA-treated (2 hours) Col-0 MYC2::MYC2-Ypet and Col-0 MYC3::MYC3-Ypet seedlings.

642 Three independent biological ChIP-seq replicates are shown. In addition, mRNA expression of

643 the three example genes Col-0 seedlings (-/+ 2 hours JA) is shown as well. Expression data is

644 derived from RNA-seq analysis. d, Venn diagram illustrates the overlap between MYC2, MYC3

645 target genes and genes that are differentially expressed after two hours of JA treatment (JA $2 \mathrm{~h}$

646 DEGs). e, f, The top-ranked motif in MYC2 (e) and MYC3 (f) ChIP-seq data was the G-box

647 (CAC/TGTG. Motifs were determined by MEME analysis using the top-ranked peaks that were

648 identified by GEM Motifs enriched in MYC2 and MYC3 peaks.

649 Figure 2. MYC2 and MYC3 target a large proportion of JA-responsive genes that encode 650 transcription factors.

651 a, b, Cluster analysis revealed the two main clusters in the JA time course experiment. The JA 652 cluster (a) with 796 genes reflects the majority of JA-induced genes and the cell wall cluster (b) 653 with 647 genes represents the largest cluster of JA repressed genes. Clusters visualize the log2 654 fold change expression dynamics over the indicated 24 hours' time period. The three strongest 655 enriched gene ontology terms for each cluster are shown as well. c, Bar plots illustrates the portion 656 of JA differentially expressed genes (JA DEGs) that are bound by MYC2 and/or MYC3 at the 657 indicated time points. JA DEGs for all time points were identified with RNA-seq. MYC2/3 targets 658 are derived from ChIP-seq analysis using Col-0 MYC2::MYC2-Ypet and Col-0 MYC3::MYC3-Ypet 
seedlings that were treated for two hours with JA. d, MYC2 and MYC3 target genes from a wide

660 range of transcription factor TF families. TF families are classified into four different groups;

661 MYC2/MYC3 targets and differentially expressed after JA treatment (blue), MYC2/MYC3 targets

662 and not differentially expressed (orange), not bound by MYC2/MYC3 but differentially expressed

663 (grey) and not bound by MYC2/MYC3 but not differentially expressed (green). e, Nodes represent

664 JA TFs for which direct binding data was generated. ChIP-seq data is indicated by presence of *,

665 all other data was DAP-seq. Edges represent binding events and are directed. Self-loops indicate

666 TF binds to its own locus, indicative of potential auto-regulation. Expression of the TF at $0.5 \mathrm{~h}$

667 after JA treatment is represented by color scale. f, Pearson correlation of TFs' target sets of

668 genes. Numerals in brackets indicate total number of target genes

\section{Figure 3. The jasmonic acid-responsive epigenome}

670 a, b, c, Aggregated profiles show the $\log _{2}$ fold change enrichment of MYC2 (a) and H3K4me3 (b,

671 c) from $2 \mathrm{~kb}$ upstream to $2 \mathrm{~kb}$ downstream of the transcriptional start site (TSS) at JA-induced

672 and JA-repressed genes. Profile of MYC2 is shown for Col-0 MYC2::MYC2-Ypet (a) seedlings

673 and H3K4me3 profiles are shown are shown for Col-0 (b) and myc2 (c) seedlings. d, AnnoJ

674 genome browser screenshot visualizes MYC2 binding, mRNA expression and H3K4me3

675 occupancy at two example genes (JAZ2, GRX480) in Col-0 and myc2 seedlings. All tracks were

676 normalized to the respective sequencing depth.

677 Figure 4. Loss of functional MYC2 impacts the global proteome and phosphoproteome

678 a, Total significantly differentially abundant $(q<0.1)$ proteins and phosphopeptides detected in

679 comparisons between JA-treated (2 h) Col-0 and myc2 seedlings and mock controls. b, Venn

680 diagram showing the overlap between significantly differentially abundant proteins, transcripts

681 and differentially phosphorylated proteins in JA-treated Col-0 seedlings compared to mock-

682 treated Col-0 controls. Also shown is the overlap with MYC2/3 target genes. c, Correlation

683 between $\log _{2}($ FPKM $)$ s of detected proteins and transcripts in Col-0 seedlings treated with JA for 
$6842 \mathrm{~h}$. Scatter plot of $\log _{2}$ fold change in Col-0 JA-regulated transcript levels versus $\log _{2}$ fold change

685 in levels of corresponding proteins. d, Heatmap represents relative TPM of 137 isoform pairs

686 exhibiting isoform switch events. Ratio calculated as logTPM (isoform 1/isoform 2). e, Plot shows

687 an example of a transcript pair originating from AT2G43680 that had isoform switch events

688 following JA treatment.

689 Figure 5. JA response genome regulatory model positions known and new components

690 a, b, Subnetworks of STZ (a) and ABO3 (b) are shown. Edges are directed. Red edges exist at 691 early time points $(0.25-2 \mathrm{~h})$, blue only at late time points $(4-24 \mathrm{~h})$. Thicker edges with chevrons

692 indicate a MYC2 directly bound that gene in ChIP-seq experiments. c, d, JA-induced root growth

693 inhibition assay identified $\mathrm{ABO} 3$ as a positive JA regulator. Seedlings were grown on LS media

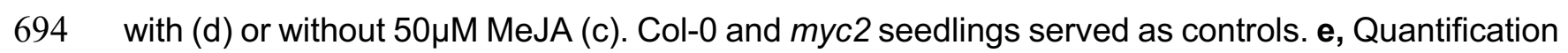

695 of JA-induced root growth inhibition in Col-0 and abo3 seedlings is shown. f, g, Root growth

696 inhibition assay identified two cyp708A2 T-DNA alleles as JA hyper-sensitive. Seedlings were

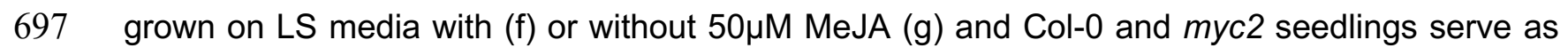

698 controls. h, Bar plot shows quantification of JA-induced root growth inhibition in Col-0 and 699 cyp708A2 seedlings.

700 Supplementary Figures

701 Supplementary Figure 1. MYC2 and MYC3 regulate the majority of JA signaling pathway 702 components

703 a, Gene ontology (GO) analysis of MYC2 and MYC3 targets is shown. Analysis was conducted 704 using clusterProfiler. b, Bar plots shows the portion of JA-induced and JA-repressed genes that 705 are bound by MYC2 (b) and MYC3 (c). c, Binding behavior of MYC2 and MYC3 at known JA 706 genes (Supplementary Table 4) is shown. Known JA genes are grouped into non-differentially 707 expressed and JA differentially expressed genes. d, Schematic overview of known MYC2/MYC3708 targeted JA pathway components. e, AnnoJ genome browser screenshot visualizes MYC2 and 
709 MYC3 binding at all 13 members of the JAZ repressor family, as well as at the co-repressor

710 adaptor protein NINJA. mRNA expression of these genes in untreated/JA-treated Col-0 and myc2

711 seedlings is also shown.

\section{Supplementary Figure 2. MYC2 and MYC3 target a large number of TFs}

713 a. Cluster analysis revealed the 5 other main clusters in the JA time course experiment. Clusters

714 visualize the log2 fold change expression dynamics over the indicated 24 hours' time period. The

715 three strongest enriched gene ontology terms for each cluster are shown as well. b, Pie Chart

716 indicates the proportions of TFs that are transcriptionally induced by JA, bound by MYC2/MYC3,

717 or both. c, d, Overview of MYC2/MYC3-bound plant hormone genes (c) and TFs (d) is shown.

718 Plant hormones are abbreviated (ET (ethylene), BR (brassinosteroids), GA (gibberellic acid), ABA

719 (abscisic acid), SA (salicylic acid), CK (cytokinin), AUX (Auxin), K (karrikin), SL (strigolactones)).

\section{Supplementary Figure 3. Overview of MYC-controlled TF network}

721 a. Significantly enriched (adjusted $p<0.05$ ) gene ontology terms amongst the target of each TF.

722 For each TF the 4 terms with the lowest $p$-value are shown, some of which are redundant between

723 TFs. No enriched terms were detected for DREB2B targets.

\section{Supplementary Figure 4. Jasmonic acid shapes the local chromatin architecture}

725 a, Bar plot shows the impact of two hours JA treatment on the genome-wide distribution of $726 \mathrm{H} 3 \mathrm{~K} 4 \mathrm{me} 3$ and H2A.Z domains. Occupancy was determined in untreated/JA-treated Col-0 and 727 myc2 seedlings using ChIP-seq. SICER was used to identify the number of histone domains that 728 show an increase (blue) or decrease (orange) of enrichment in response to JA. b, c, Heatmaps 729 show the occupancy of $\mathrm{H} 3 \mathrm{~K} 4 \mathrm{me} 3$ and H2A.Z from $1 \mathrm{~kb}$ upstream to $2 \mathrm{~kb}$ downstream of the 730 transcriptional start site (TSS) at all Arabidopsis genes (TAIR10). Heatmaps are shown for $731 \mathrm{H} 3 \mathrm{~K} 4 \mathrm{me} 3(\mathrm{~b})$ and H2A.Z (c) in untreated and JA-treated (4 h) Col-0 and myc2 seedlings. d, 732 Quantification of H3K4me3 and H2A.Z occupancy at JAZ2 and GRX480 are shown. It was 733 calculated as the ratio between the respective ChIP-seq sample and the Col-0 IgG control. 


\section{Supplementary Figure 5. The jasmonic acid gene regulatory network}

735 a, Illustration of JA gene regulatory network for 1, 2 and $4 \mathrm{~h}$ time points. Edges were predicted 736 using phosphoproteome (green), proteome (orange) and transcriptome (blue) data. Node sizes

737 are scaled by normalized motif score, with larger nodes indicating greater scores and likely

738 greater importance within the network. Edges predicted early in the time-series transcriptomic

739 data are red $(0.25-2 \mathrm{~h})$, edges predicted late are blue $(4-24 \mathrm{~h})$. Proteome and

740 phosphoproteome-data-predicted edges are grey and green, respectively.

741 Supplementary Figure 6. Gene regulatory network validation against ChIP/DAP-seq data a,

742 The MYC2 subnetwork is shown. Edges are directional and red edges exist at early time points

$743(0.25-2 \mathrm{~h})$, blue only at late time points $(4-24 \mathrm{~h})$. Thicker edges with chevrons indicate that

744 MYC2 were directly bound to that gene in our ChIP-seq experiments. b, Validated edges are

745 those between TFs and first neighbours in the JA gene regulatory network for which the first 746 neighbour was also a direct target of the TF in ChIP/DAP-seq assays. These edges are indicated

747 by chevrons. Early time-series transcriptome-predicted edges $(0.25-2 \mathrm{~h})$ are red and later edges

$748(4-24 \mathrm{~h})$ are blue. Edges detected in the proteomic data are grey and those detected in the 749 phosphoproteomic data are green.

\section{Supplementary Figure 7 Validation of regulatory network predictions}

751 a, Bar plot shows quantification of JA-induced root growth inhibition in the indicated T-DNA alleles.

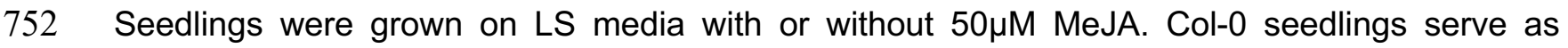

753 independent controls for each tested T-DNA line. b, Subnetwork of CYP708A2 is shown.

\section{Supplementary Tables}

755 Supplementary Table 1. High-confidence target genes of MYC2 and MYC3 after two hours JA 756 treatment. Unprocessed summit outputs of biological replicate experiments including $p$-value 757 scores. Hormone transcription factors bound by MYC2/3. 
758 Supplementary Table 2. Differential regulation of all transcripts relative to $0 \mathrm{~h}$ abundance

759 following JA treatment. Tab names indicate time point post-treatment. Calculated by EdgeR with

760 false discovery rate $($ FDR $)<0.05$ indicating statistical significance. FC - fold change. CPM - counts

761 per million. LR - likelihood ratio.

762 Supplementary Table 3. Motifs detected de novo within MYC2 and MYC3 target summits. The

763 data are DREME model outputs for MYC2 and MYC3 high-stringency summits.

764 Supplementary Table 4. Expression of 168 known JA genes following JA stimulus and whether

765 they are bound by $\mathrm{MYC} 2 / 3$ or not. ND indicates non-differentially regulated, as assessed by

766 EdgeR (FDR<0.05).

767 Supplementary Table 5. Details of STEM model of JA-responsive transcripts and details of 768 transcripts within statistically significant clusters. Input data were the expression values of all 769 transcripts significantly differentially regulated at any time in the time series relative to $0 \mathrm{~h}$ post770 JA stimulus.

771 Supplementary Table 6. Target genes of JA transcription factors identified by ChIP-seq 772 (NAC3/ANAC055, STZ/ZAT10; indicated by *) and DAP-seq (DREB2B, ATAF2, HY5, RVE2, 773 ZAT18, TCP23, ERF1, ORA59, WRKY51).

774 Supplementary Table 7. Differentially enriched H3K4me3 and H2A.Z domains in JA-treated Col7750 and myc2 seedlings.

776 Supplementary Table 8. Differentially expressed proteins detected in proteomics analyses.

777 Supplementary Table 9. Differentially abundant phosphopeptides detected in phosphoproteomic 778 analyses.

779 Supplementary Table 10. Transcript pairs exhibiting isoform switch events as detected by TSIS 780 analyses.

781 Supplementary Table 11. TPM quantification of transcripts in the JA time-series RNA-seq 782 against the AtRD2 reference transcriptome. 
Supplementary Table 12. Nodes and edges within the JA response genome regulatory network model, generated from combined JA (phospho)proteome and transcriptome data. Normalized motif score of all components is also given.

Supplementary Table 13. Gene regulatory network validation against ChIP/DAP-seq data. Validated edges are those between TFs and first neighbours in the JA gene regulatory network for which the first neighbour was also a direct target of the TF in ChIP/DAP-seq assays. * indicates ChIP-seq assay, all others were DAP-seq.

Supplementary Table 14. List of tested T-DNA lines and T-DNA lines with a JA root growth 791 inhibition phenotypes.

792 Supplementary Table 15. List of parameters used during gene regulatory network 793 reconstruction.

References

795

(2000). Analysis of the genome sequence of the flowering plant Arabidopsis thaliana. Nature, 408, 796-815.

Abe, H., Urao, T., Ito, T., Seki, M., Shinozaki, K. and Yamaguchi-Shinozaki, K. (2003). Arabidopsis AtMYC2 (bHLH) and AtMYB2 (MYB) function as transcriptional activators in abscisic acid signaling. The Plant cell, 15, 63-78.

Aleman, F., Yazaki, J., Lee, M., Takahashi, Y., Kim, A. Y., Li, Z., Kinoshita, T., Ecker, J. R. and Schroeder, J. I. (2016). An ABA-increased interaction of the PYL6 ABA receptor with MYC2 Transcription Factor: A putative link of ABA and JA signaling. Scientific reports, 6 , 28941.

Alon, U. (2007). Network motifs: theory and experimental approaches. Nature reviews. Genetics, 8, 450-61.

An, C., et al. (2017). Mediator subunit MED25 links the jasmonate receptor to transcriptionally active chromatin. Proceedings of the National Academy of Sciences of the United States of America, 114, E8930-E8939.

An, F., et al. (2010). Ethylene-induced stabilization of ETHYLENE INSENSITIVE3 and EIN3LIKE1 is mediated by proteasomal degradation of EIN3 binding F-box 1 and 2 that requires EIN2 in Arabidopsis. The Plant cell, 22, 2384-401.

Anders, S., Pyl, P. T. and Huber, W. (2015). HTSeq--a Python framework to work with highthroughput sequencing data. Bioinformatics, 31, 166-9.

Baerenfaller, K., et al. (2008). Genome-scale proteomics reveals Arabidopsis thaliana gene models and proteome dynamics. Science, 320, 938-41.

Bao, S., Hua, C., Huang, G., Cheng, P., Gong, X., Shen, L. and Yu, H. (2019). Molecular Basis of Natural Variation in Photoperiodic Flowering Responses. Developmental cell.

Bartlett, A., O'malley, R. C., Huang, S. C., Galli, M., Nery, J. R., Gallavotti, A. and Ecker, J. R. (2017). Mapping genome-wide transcription-factor binding sites using DAP-seq. Nature protocols, 12, 1659-1672. 
821

822

823

824

825

826

827

828

829

830

831

832

833

834

835

836

837

838

839

840

841

842

843

844

845

846

847

848

849

850

851

852

853

854

855

856

857

858

859

860

861

862

863

864

865

866

867

868

869

870

871

Bu, Q., Jiang, H., Li, C. B., Zhai, Q., Zhang, J., Wu, X., Sun, J., Xie, Q. and Li, C. (2008). Role of the Arabidopsis thaliana NAC transcription factors ANAC019 and ANAC055 in regulating jasmonic acid-signaled defense responses. Cell research, 18, 756-67.

Calixto, C. P. G., et al. (2018). Rapid and Dynamic Alternative Splicing Impacts the Arabidopsis Cold Response Transcriptome. The Plant cell, 30, 1424-1444.

Chang, K. N., et al. (2013). Temporal transcriptional response to ethylene gas drives growth hormone cross-regulation in Arabidopsis. eLife, 2, e00675.

Chen, H. Y., Hsieh, E. J., Cheng, M. C., Chen, C. Y., Hwang, S. Y. and Lin, T. P. (2016a). ORA47 (octadecanoid-responsive AP2/ERF-domain transcription factor 47) regulates jasmonic acid and abscisic acid biosynthesis and signaling through binding to a novel cis-element. The New phytologist, 211, 599-613.

Chen, X., Huang, H., Qi, T., Liu, B. and Song, S. (2016b). New perspective of the bHLH-MYB complex in jasmonate-regulated plant fertility in arabidopsis. Plant signaling \& behavior, $11, \mathrm{e} 1135280$.

Chini, A., et al. (2007). The JAZ family of repressors is the missing link in jasmonate signalling. Nature, 448, 666-71.

Chung, H. S., Cooke, T. F., Depew, C. L., Patel, L. C., Ogawa, N., Kobayashi, Y. and Howe, G. A. (2010). Alternative splicing expands the repertoire of dominant JAZ repressors of jasmonate signaling. The Plant journal : for cell and molecular biology, 63, 613-22.

Coleman-Derr, D. and Zilberman, D. (2012). Deposition of histone variant H2A.Z within gene bodies regulates responsive genes. PLoS genetics, 8, e1002988.

Cox, J., Neuhauser, N., Michalski, A., Scheltema, R. A., Olsen, J. V. and Mann, M. (2011). Andromeda: a peptide search engine integrated into the MaxQuant environment. Journal of proteome research, 10, 1794-805.

Cuellar Perez, A., et al. (2014). The non-JAZ TIFY protein TIFY8 from Arabidopsis thaliana is a transcriptional repressor. PloS one, 9, e84891.

Cui, H., Quu, J., Zhou, Y., Bhandari, D. D., Zhao, C., Bautor, J. and Parker, J. E. (2018). Antagonism of Transcription Factor MYC2 by EDS1/PAD4 Complexes Bolsters Salicylic Acid Defense in Arabidopsis Effector-Triggered Immunity. Molecular plant, 11, 1053-1066.

Du, M., et al. (2017). MYC2 Orchestrates a Hierarchical Transcriptional Cascade That Regulates Jasmonate-Mediated Plant Immunity in Tomato. The Plant cell, 29, 1883-1906.

Dubois, M., Van Den Broeck, L., Claeys, H., Van Vlierberghe, K., Matsui, M. and Inze, D. (2015). The ETHYLENE RESPONSE FACTORs ERF6 and ERF11 Antagonistically Regulate Mannitol-Induced Growth Inhibition in Arabidopsis. Plant physiology, 169, 166-79.

Elias, J. E. and Gygi, S. P. (2010). Target-decoy search strategy for mass spectrometry-based proteomics. Methods in molecular biology, 604, 55-71.

Ernst, J. and Bar-Joseph, Z. (2006). STEM: a tool for the analysis of short time series gene expression data. BMC bioinformatics, 7, 191.

Feng, S., Jacobsen, S. E. and Reik, W. (2010). Epigenetic reprogramming in plant and animal development. Science, 330, 622-7.

Fernandez-Calvo, P., et al. (2011). The Arabidopsis bHLH transcription factors MYC3 and MYC4 are targets of JAZ repressors and act additively with MYC2 in the activation of jasmonate responses. The Plant cell, 23, 701-15.

Fernandez, P. C., Frank, S. R., Wang, L., Schroeder, M., Liu, S., Greene, J., Cocito, A. and Amati, B. (2003). Genomic targets of the human c-Myc protein. Genes \& development, 17, 111529.

Field, B. and Osbourn, A. E. (2008). Metabolic diversification--independent assembly of operonlike gene clusters in different plants. Science, 320, 543-7.

Fonseca, S., Chini, A., Hamberg, M., Adie, B., Porzel, A., Kramell, R., Miersch, O., Wasternack, C. and Solano, R. (2009). (+)-7-iso-Jasmonoyl-L-isoleucine is the endogenous bioactive jasmonate. Nature chemical biology, 5, 344-50. 
872

873

874

875

876

877

878

879

880

881

882

883

884

885

886

887

888

889

890

891

892

893

894

895

896

897

898

899

900

901

902

903

904

905

906

907

908

909

910

911

912

913

914

915

916

917

918

919

920

921

Fonseca, S., et al. (2014). bHLH003, bHLH013 and bHLH017 are new targets of JAZ repressors negatively regulating JA responses. PloS one, 9, e86182.

Gao, Q. M., Venugopal, S., Navarre, D. and Kachroo, A. (2011). Low oleic acid-derived repression of jasmonic acid-inducible defense responses requires the WRKY50 and WRKY51 proteins. Plant physiology, 155, 464-76.

Gimenez-Ibanez, S., Boter, M., Ortigosa, A., Garcia-Casado, G., Chini, A., Lewsey, M. G., Ecker, J. R., Ntoukakis, V. and Solano, R. (2017). JAZ2 controls stomata dynamics during bacterial invasion. The New phytologist, 213, 1378-1392.

Godoy, M., Franco-Zorrilla, J. M., Perez-Perez, J., Oliveros, J. C., Lorenzo, O. and Solano, R. (2011). Improved protein-binding microarrays for the identification of DNA-binding specificities of transcription factors. The Plant journal : for cell and molecular biology, 66, 700-11.

Guo, Q., Yoshida, Y., Major, I. T., Wang, K., Sugimoto, K., Kapali, G., Havko, N. E., Benning, C. and Howe, G. A. (2018). JAZ repressors of metabolic defense promote growth and reproductive fitness in Arabidopsis. Proceedings of the National Academy of Sciences of the United States of America, 115, E10768-E10777.

Guo, W., Calixto, C. P. G., Brown, J. W. S. and Zhang, R. (2017). TSIS: an R package to infer alternative splicing isoform switches for time-series data. Bioinformatics, 33, 3308-3310.

Guo, Y., Mahony, S. and Gifford, D. K. (2012). High resolution genome wide binding event finding and motif discovery reveals transcription factor spatial binding constraints. PLoS computational biology, 8, e1002638.

Hartmann, L., et al. (2016). Alternative Splicing Substantially Diversifies the Transcriptome during Early Photomorphogenesis and Correlates with the Energy Availability in Arabidopsis. The Plant cell, 28, 2715-2734.

Hentrich, M., Bottcher, C., Duchting, P., Cheng, Y., Zhao, Y., Berkowitz, O., Masle, J., Medina, J. and Pollmann, S. (2013). The jasmonic acid signaling pathway is linked to auxin homeostasis through the modulation of YUCCA8 and YUCCA9 gene expression. The Plant journal : for cell and molecular biology, 74, 626-37.

Hickman, R., et al. (2017). Architecture and Dynamics of the Jasmonic Acid Gene Regulatory Network. The Plant cell, 29, 2086-2105.

Hou, X., Lee, L. Y., Xia, K., Yan, Y. and Yu, H. (2010). DELLAs modulate jasmonate signaling via competitive binding to JAZs. Developmental cell, 19, 884-94.

Huang, H., Liu, B., Liu, L. and Song, S. (2017). Jasmonate action in plant growth and development. Journal of experimental botany, 68, 1349-1359.

Huang, J., Ghosh, R. and Bankaitis, V. A. (2016). Sec14-like phosphatidylinositol transfer proteins and the biological landscape of phosphoinositide signaling in plants. Biochimica et biophysica acta, 1861, 1352-1364.

Huot, B., Yao, J., Montgomery, B. L. and He, S. Y. (2014). Growth-defense tradeoffs in plants: a balancing act to optimize fitness. Molecular plant, 7, 1267-1287.

Ingram, P. J., Stumpf, M. P. and Stark, J. (2006). Network motifs: structure does not determine function. BMC genomics, 7, 108.

Jin, J., Tian, F., Yang, D. C., Meng, Y. Q., Kong, L., Luo, J. and Gao, G. (2017). PlantTFDB 4.0: toward a central hub for transcription factors and regulatory interactions in plants. Nucleic acids research, 45, D1040-D1045.

Kang, H. G., Kim, J., Kim, B., Jeong, H., Choi, S. H., Kim, E. K., Lee, H. Y. and Lim, P. O. (2011). Overexpression of FTL1/DDF1, an AP2 transcription factor, enhances tolerance to cold, drought, and heat stresses in Arabidopsis thaliana. Plant science : an international journal of experimental plant biology, 180, 634-41.

Karasov, T. L., Chae, E., Herman, J. J. and Bergelson, J. (2017). Mechanisms to Mitigate the Trade-Off between Growth and Defense. The Plant cell, 29, 666-680. 
922

923

924

925

926

927

928

929

930

931

932

933

934

935

936

937

938

939

940

941

942

943

944

945

946

947

948

949

950

951

952

953

954

955

956

957

958

959

960

961

962

963

964

965

966

967

968

969

970

971

972

Kaufmann, K., Muino, J. M., Osteras, M., Farinelli, L., Krajewski, P. and Angenent, G. C. (2010). Chromatin immunoprecipitation (ChIP) of plant transcription factors followed by sequencing (ChIP-SEQ) or hybridization to whole genome arrays (ChIP-CHIP). Nature protocols, 5, 457-72.

Khan, A. and Mathelier, A. (2017). Intervene: a tool for intersection and visualization of multiple gene or genomic region sets. BMC bioinformatics, 18, 287.

Kharchenko, P. V., Tolstorukov, M. Y. and Park, P. J. (2008). Design and analysis of ChIP-seq experiments for DNA-binding proteins. Nature biotechnology, 26, 1351-9.

Kim, D., Pertea, G., Trapnell, C., Pimentel, H., Kelley, R. and Salzberg, S. L. (2013). TopHat2: accurate alignment of transcriptomes in the presence of insertions, deletions and gene fusions. Genome biology, 14, R36.

Langmead, B. (2010). Aligning short sequencing reads with Bowtie. Current protocols in bioinformatics, Chapter 11, Unit 117.

Langmead, B. and Salzberg, S. L. (2012). Fast gapped-read alignment with Bowtie 2. Nature methods, 9, 357-9.

Li, J., Brader, G., Kariola, T. and Palva, E. T. (2006). WRKY70 modulates the selection of signaling pathways in plant defense. The Plant journal : for cell and molecular biology, 46, 477-91.

Liu, Y., et al. (2019). MYC2 Regulates the Termination of Jasmonate Signaling via an Autoregulatory Negative Feedback Loop. The Plant cell, 31, 106-127.

Lorenzo, O., Chico, J. M., Sanchez-Serrano, J. J. and Solano, R. (2004). JASMONATEINSENSITIVE1 encodes a MYC transcription factor essential to discriminate between different jasmonate-regulated defense responses in Arabidopsis. The Plant cell, 16, 193850 .

Lorenzo, O., Piqueras, R., Sanchez-Serrano, J. J. and Solano, R. (2003). ETHYLENE RESPONSE FACTOR1 integrates signals from ethylene and jasmonate pathways in plant defense. The Plant cell, 15, 165-78.

Lozano-Duran, R., Macho, A. P., Boutrot, F., Segonzac, C., Somssich, I. E. and Zipfel, C. (2013). The transcriptional regulator BZR1 mediates trade-off between plant innate immunity and growth. eLife, 2, e00983.

Lun, A. T., Chen, Y. and Smyth, G. K. (2016). It's DE-licious: A Recipe for Differential Expression Analyses of RNA-seq Experiments Using Quasi-Likelihood Methods in edgeR. Methods in molecular biology, 1418, 391-416.

Machanick, P. and Bailey, T. L. (2011). MEME-ChIP: motif analysis of large DNA datasets. Bioinformatics, 27, 1696-7.

Magome, H., Yamaguchi, S., Hanada, A., Kamiya, Y. and Oda, K. (2008). The DDF1 transcriptional activator upregulates expression of a gibberellin-deactivating gene, GA2ox7, under high-salinity stress in Arabidopsis. The Plant journal : for cell and molecular biology, 56, 613-26.

Mangas, S., Bonfill, M., Osuna, L., Moyano, E., Tortoriello, J., Cusido, R. M., Pinol, M. T. and Palazon, J. (2006). The effect of methyl jasmonate on triterpene and sterol metabolisms of Centella asiatica, Ruscus aculeatus and Galphimia glauca cultured plants. Phytochemistry, 67, 2041-9.

Milo, R., Shen-Orr, S., Itzkovitz, S., Kashtan, N., Chklovskii, D. and Alon, U. (2002). Network motifs: simple building blocks of complex networks. Science, 298, 824-7.

Mittler, R., Kim, Y., Song, L., Coutu, J., Coutu, A., Ciftci-Yilmaz, S., Lee, H., Stevenson, B. and Zhu, J. K. (2006). Gain- and loss-of-function mutations in Zat10 enhance the tolerance of plants to abiotic stress. FEBS letters, 580, 6537-42.

Moreno, J. E., Shyu, C., Campos, M. L., Patel, L. C., Chung, H. S., Yao, J., He, S. Y. and Howe, G. A. (2013). Negative feedback control of jasmonate signaling by an alternative splice variant of JAZ10. Plant physiology, 162, 1006-17. 
973

974

975

976

977

978

979

980

981

982

983

984

985

986

987

988

989

990

991

992

993

994

995

996

997

998

999

1000

1001

1002

1003

1004

1005

1006

1007

1008

1009

1010

1011

1012

1013

1014

1015

1016

1017

1018

1019

1020

1021

1022

1023

Mosblech, A., Thurow, C., Gatz, C., Feussner, I. and Heilmann, I. (2011). Jasmonic acid perception by COI 1 involves inositol polyphosphates in Arabidopsis thaliana. The Plant journal : for cell and molecular biology, 65, 949-57.

Nakata, M., Mitsuda, N., Herde, M., Koo, A. J., Moreno, J. E., Suzuki, K., Howe, G. A. and OhmeTakagi, M. (2013). A bHLH-type transcription factor, ABA-INDUCIBLE BHLH-TYPE TRANSCRIPTION FACTOR/JA-ASSOCIATED MYC2-LIKE1, acts as a repressor to negatively regulate jasmonate signaling in arabidopsis. The Plant cell, 25, 1641-56.

Ndamukong, I., Abdallat, A. A., Thurow, C., Fode, B., Zander, M., Weigel, R. and Gatz, C. (2007). SA-inducible Arabidopsis glutaredoxin interacts with TGA factors and suppresses JAresponsive PDF1.2 transcription. The Plant journal : for cell and molecular biology, 50, 128-39.

O'malley, R. C., Huang, S. S., Song, L., Lewsey, M. G., Bartlett, A., Nery, J. R., Galli, M., Gallavotti, A. and Ecker, J. R. (2016). Cistrome and Epicistrome Features Shape the Regulatory DNA Landscape. Cell, 165, 1280-92.

Patro, R., Duggal, G., Love, M. I., Irizarry, R. A. and Kingsford, C. (2017). Salmon provides fast and bias-aware quantification of transcript expression. Nature methods, 14, 417-419.

Pauwels, L., et al. (2010). NINJA connects the co-repressor TOPLESS to jasmonate signalling. Nature, 464, 788-91.

Pauwels, L. and Goossens, A. (2008). Fine-tuning of early events in the jasmonate response. Plant signaling \& behavior, 3, 846-7.

Pauwels, L., Morreel, K., De Witte, E., Lammertyn, F., Van Montagu, M., Boerjan, W., Inze, D. and Goossens, A. (2008). Mapping methyl jasmonate-mediated transcriptional reprogramming of metabolism and cell cycle progression in cultured Arabidopsis cells. Proceedings of the National Academy of Sciences of the United States of America, 105, $1380-5$.

Pieterse, C. M., Leon-Reyes, A., Van Der Ent, S. and Van Wees, S. C. (2009). Networking by small-molecule hormones in plant immunity. Nature chemical biology, 5, 308-16.

Pre, M., Atallah, M., Champion, A., De Vos, M., Pieterse, C. M. and Memelink, J. (2008). The AP2/ERF domain transcription factor ORA59 integrates jasmonic acid and ethylene signals in plant defense. Plant physiology, 147, 1347-57.

Quinlan, A. R. and Hall, I. M. (2010). BEDTools: a flexible suite of utilities for comparing genomic features. Bioinformatics, 26, 841-2.

Ren, X., Chen, Z., Liu, Y., Zhang, H., Zhang, M., Liu, Q., Hong, X., Zhu, J. K. and Gong, Z. (2010). ABO3, a WRKY transcription factor, mediates plant responses to abscisic acid and drought tolerance in Arabidopsis. The Plant journal : for cell and molecular biology, 63, 417-29.

Robinson, M. D., Mccarthy, D. J. and Smyth, G. K. (2010). edgeR: a Bioconductor package for differential expression analysis of digital gene expression data. Bioinformatics, 26, 13940.

Rothbart, S. B. and Strahl, B. D. (2014). Interpreting the language of histone and DNA modifications. Biochimica et biophysica acta.

Sasaki-Sekimoto, Y., Jikumaru, Y., Obayashi, T., Saito, H., Masuda, S., Kamiya, Y., Ohta, H. and Shirasu, K. (2013). Basic helix-loop-helix transcription factors JASMONATEASSOCIATED MYC2-LIKE1 (JAM1), JAM2, and JAM3 are negative regulators of jasmonate responses in Arabidopsis. Plant physiology, 163, 291-304.

Schweizer, F., Bodenhausen, N., Lassueur, S., Masclaux, F. G. and Reymond, P. (2013a). Differential Contribution of Transcription Factors to Arabidopsis thaliana Defense Against Spodoptera littoralis. Frontiers in plant science, 4, 13.

Schweizer, F., et al. (2013b). Arabidopsis basic helix-loop-helix transcription factors MYC2, MYC3, and MYC4 regulate glucosinolate biosynthesis, insect performance, and feeding behavior. The Plant cell, 25, 3117-32. 
1024

1025

1026

1027

1028

1029

1030

1031

1032

1033

1034

1035

1036

1037

1038

1039

1040

1041

1042

1043

1044

1045

1046

1047

1048

1049

1050

1051

1052

1053

1054

1055

1056

1057

1058

1059

1060

1061

1062

1063

1064

1065

1066

1067

1068

1069

1070

1071

1072

1073

Sheard, L. B., et al. (2010). Jasmonate perception by inositol-phosphate-potentiated COI1-JAZ co-receptor. Nature, 468, 400-5.

Shibata, M., et al. (2018). GTL1 and DF1 regulate root hair growth through transcriptional repression of ROOT HAIR DEFECTIVE 6-LIKE 4 in Arabidopsis. Development, 145.

Shyu, C., et al. (2012). JAZ8 lacks a canonical degron and has an EAR motif that mediates transcriptional repression of jasmonate responses in Arabidopsis. The Plant cell, 24, 53650 .

Song, L., et al. (2016). A transcription factor hierarchy defines an environmental stress response network. Science, 354.

Song, S., Huang, H., Wang, J., Liu, B., Qi, T. and Xie, D. (2017). MYC5 is Involved in JasmonateRegulated Plant Growth, Leaf Senescence and Defense Responses. Plant \& cell physiology, 58, 1752-1763.

Thines, B., et al. (2007). JAZ repressor proteins are targets of the SCF(COI1) complex during jasmonate signalling. Nature, 448, 661-5.

Tyanova, S., Temu, T. and Cox, J. (2016). The MaxQuant computational platform for mass spectrometry-based shotgun proteomics. Nature protocols, 11, 2301-2319.

Van Aken, O., Zhang, B., Law, S., Narsai, R. and Whelan, J. (2013). AtWRKY40 and AtWRKY63 modulate the expression of stress-responsive nuclear genes encoding mitochondrial and chloroplast proteins. Plant physiology, 162, 254-71.

Vanstraelen, M. and Benkova, E. (2012). Hormonal interactions in the regulation of plant development. Annual review of cell and developmental biology, 28, 463-87.

Walley, J. W., et al. (2016). Integration of omic networks in a developmental atlas of maize. Science, 353, 814-8.

Wang, C., Liu, Y., Li, S. S. and Han, G. Z. (2015). Insights into the origin and evolution of the plant hormone signaling machinery. Plant physiology, 167, 872-86.

Wang, H., et al. (2019). MED25 connects enhancer-promoter looping and MYC2-dependent activation of jasmonate signalling. Nature plants, 5, 616-625.

Wild, M., Daviere, J. M., Cheminant, S., Regnault, T., Baumberger, N., Heintz, D., Baltz, R., Genschik, P. and Achard, P. (2012). The Arabidopsis DELLA RGA-LIKE3 is a direct target of MYC2 and modulates jasmonate signaling responses. The Plant cell, 24, 3307-19.

Xiao, J., Jin, R. and Wagner, D. (2017). Developmental transitions: integrating environmental cues with hormonal signaling in the chromatin landscape in plants. Genome biology, 18, 88.

Xie, D. X., Feys, B. F., James, S., Nieto-Rostro, M. and Turner, J. G. (1998). COI1: an Arabidopsis gene required for jasmonate-regulated defense and fertility. Science, 280, 1091-4.

Xie, M., Chen, H., Huang, L., O'neil, R. C., Shokhirev, M. N. and Ecker, J. R. (2018). A B-ARRmediated cytokinin transcriptional network directs hormone cross-regulation and shoot development. Nature communications, 9, 1604.

Xu, J., Li, Y., Wang, Y., Liu, H., Lei, L., Yang, H., Liu, G. and Ren, D. (2008). Activation of MAPK kinase 9 induces ethylene and camalexin biosynthesis and enhances sensitivity to salt stress in Arabidopsis. The Journal of biological chemistry, 283, 26996-7006.

Yu, G., Wang, L. G., Han, Y. and He, Q. Y. (2012). clusterProfiler: an R package for comparing biological themes among gene clusters. Omics : a journal of integrative biology, 16, 2847.

Zander, M., Chen, S., Imkampe, J., Thurow, C. and Gatz, C. (2012). Repression of the Arabidopsis thaliana jasmonic acid/ethylene-induced defense pathway by TGA-interacting glutaredoxins depends on their C-terminal ALWL motif. Molecular plant, 5, 831-40.

Zang, C., Schones, D. E., Zeng, C., Cui, K., Zhao, K. and Peng, W. (2009). A clustering approach for identification of enriched domains from histone modification ChIP-Seq data. Bioinformatics, 25, 1952-8. 
1074

1075

1076

1077

1078

1079

1080

1081

1082

1083

1084

1085

1086

1087

1088

1089

1090

1091

1092

1093
Zhang, F., et al. (2017). Structural insights into alternative splicing-mediated desensitization of jasmonate signaling. Proceedings of the National Academy of Sciences of the United States of America, 114, 1720-1725.

Zhang, F., et al. (2015a). Structural basis of JAZ repression of MYC transcription factors in jasmonate signalling. Nature, 525, 269-73.

Zhang, R., et al. (2015b). AtRTD - a comprehensive reference transcript dataset resource for accurate quantification of transcript-specific expression in Arabidopsis thaliana. The New phytologist, 208, 96-101.

Zhang, X., Zhu, Z., An, F., Hao, D., Li, P., Song, J., Yi, C. and Guo, H. (2014). Jasmonateactivated MYC2 represses ETHYLENE INSENSITIVE3 activity to antagonize ethylenepromoted apical hook formation in Arabidopsis. The Plant cell, 26, 1105-17.

Zhang, Y., et al. (2008). Model-based analysis of ChIP-Seq (MACS). Genome biology, 9, R137.

Zhou, R., Benavente, L. M., Stepanova, A. N. and Alonso, J. M. (2011). A recombineering-based gene tagging system for Arabidopsis. The Plant journal : for cell and molecular biology, 66, 712-23.

Zhu, L. J., Gazin, C., Lawson, N. D., Pages, H., Lin, S. M., Lapointe, D. S. and Green, M. R. (2010). ChIPpeakAnno: a Bioconductor package to annotate ChIP-seq and ChIP-chip data. BMC bioinformatics, 11, 237. 
bio:axiv preprint doi: https://doi.org/10.1101/736363; this version posted August 19,2019 . The copyright holder for this preprint (which was not Figureertilied by peer review) is the author/funder, who has granted bioRxiv a license to display the preprint in perpetuity. It is made available under

a

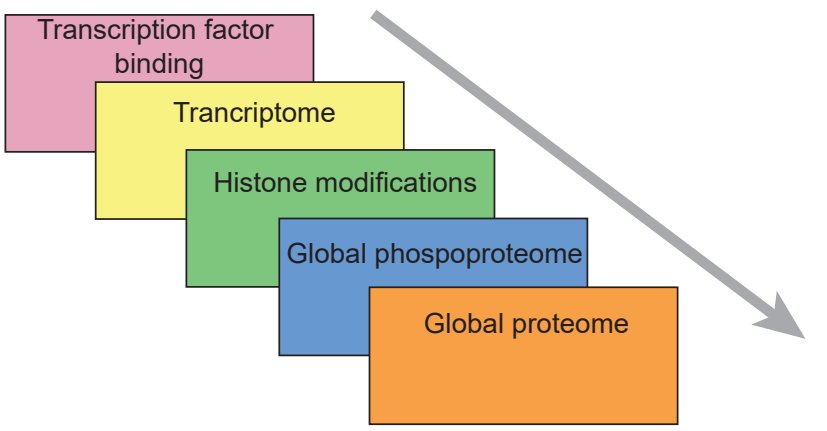

C

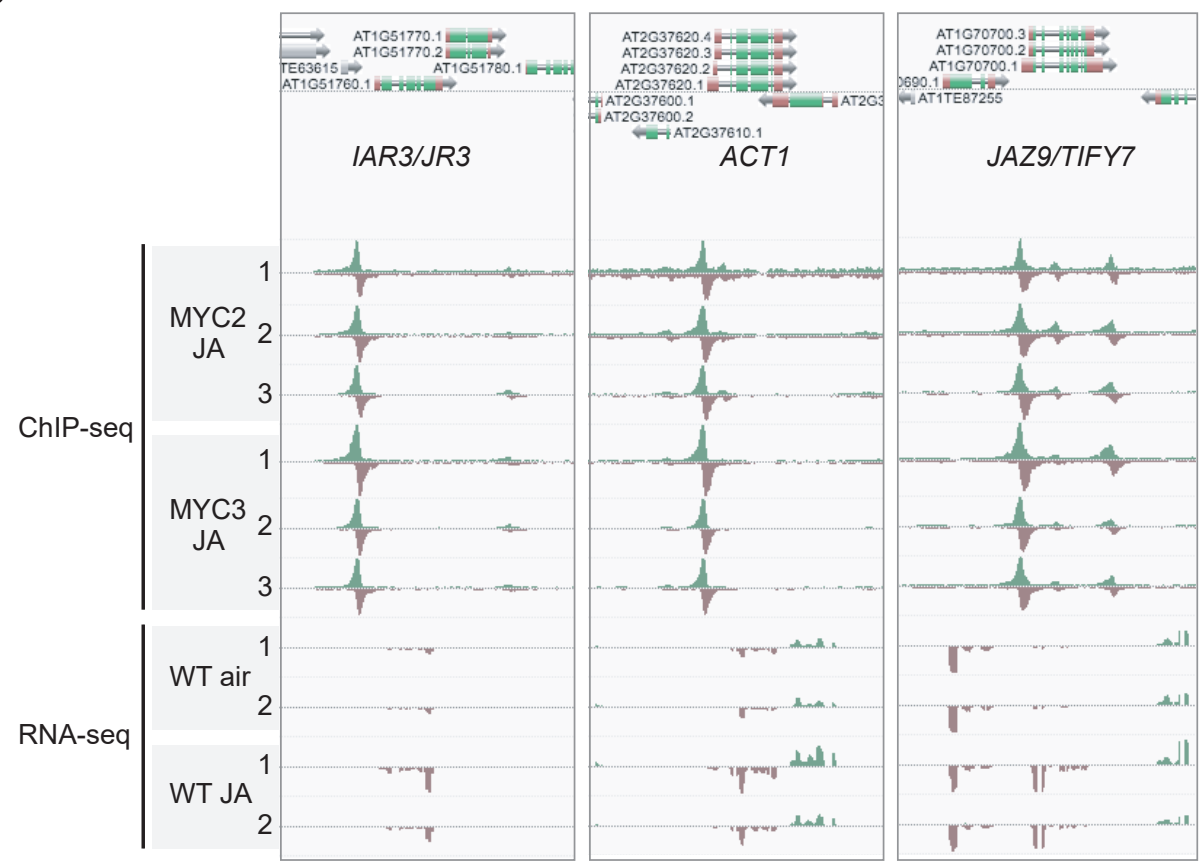

b

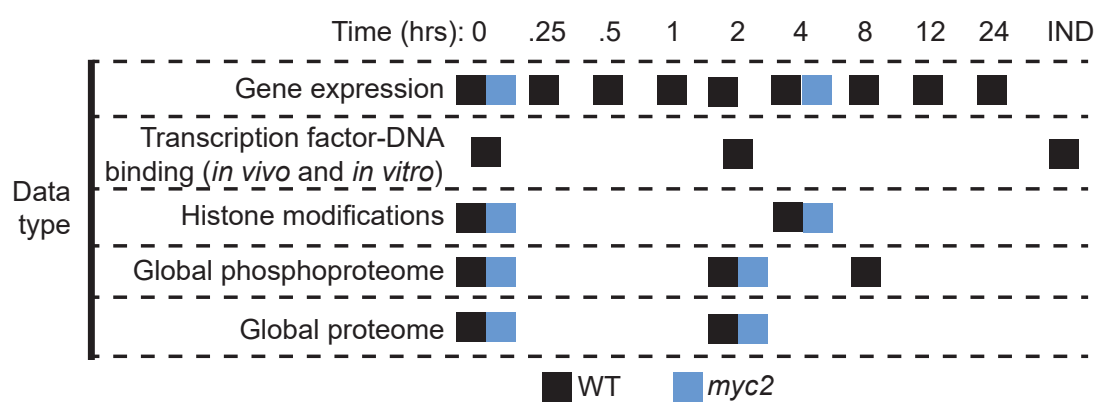

d

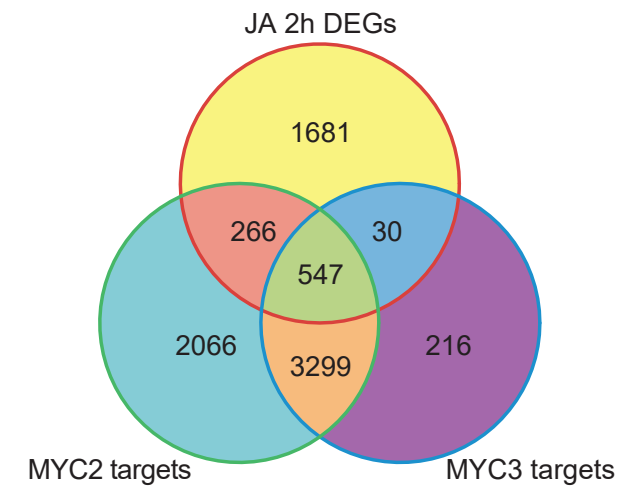

e

MYC2

f

MYC3

4240 of 6736 targets

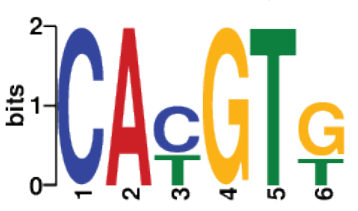

3072 of 3982 targets

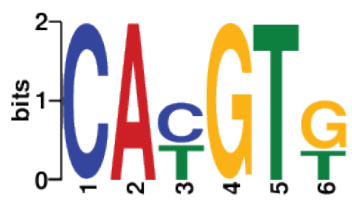


bioRyiv preprint doi: https://doi.org/10.1101/736363; this version posted August 19,2019 . The copyright holder for this preprint (which was not Fig U rertila by peer review) is the author/funder, who has granted bioRxiv a license to display the preprint in perpetuity. It is made available under

a

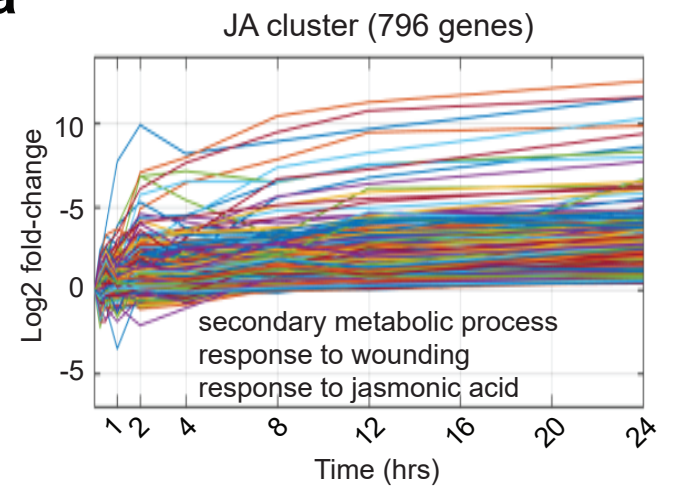

d

e

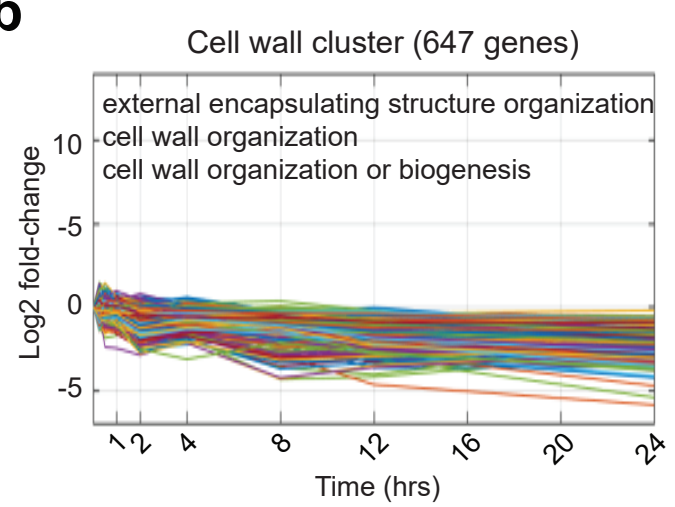

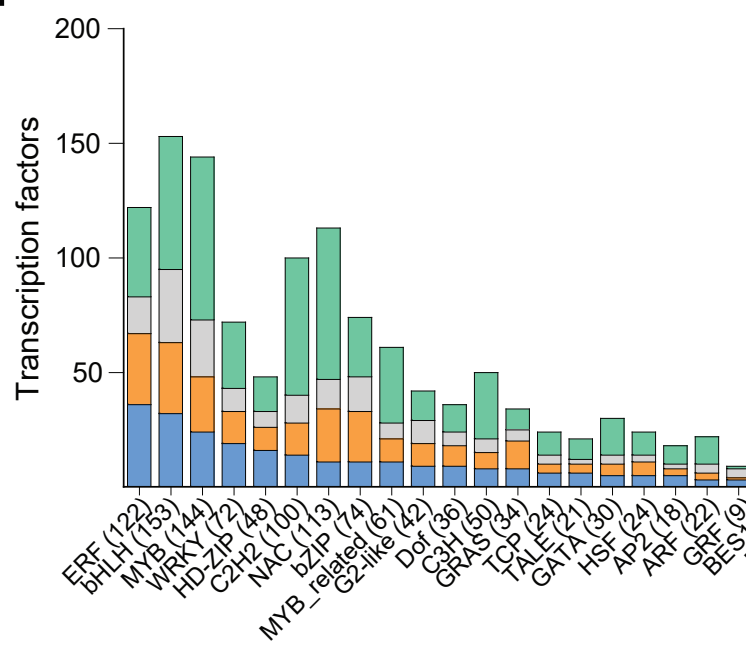

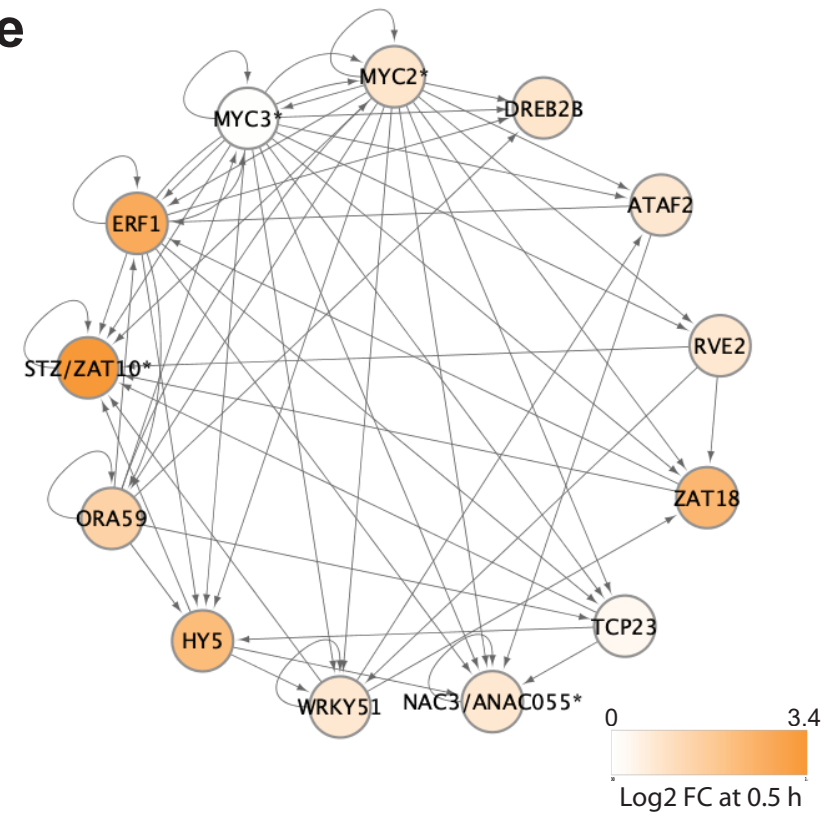

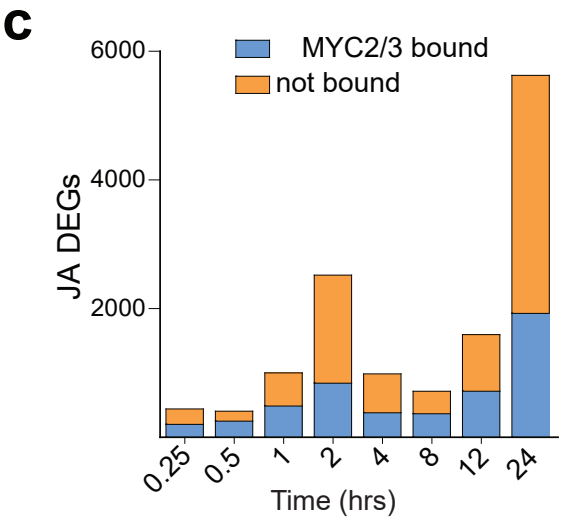

MYC2/3 target, differentially expressed MYC2/3 target, non-differentially expressed non-target, differentially expressed non-target, non-differentially expressed aCC-BY-NC-ND 4.0 International license.

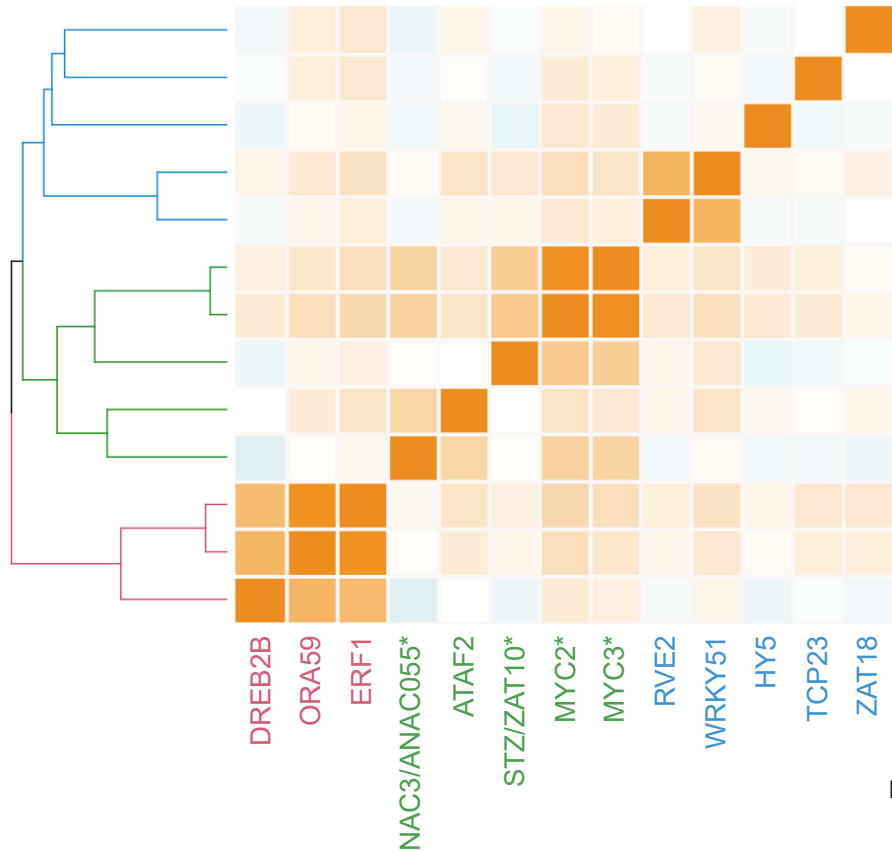

ZAT18 $(3,318)$

TCP23 $(1,732)$

HY5 $(1,068)$

WRKY51 $(6,019)$

RVE2 $(2,060)$

MYC3* $^{*}(4,092)$

MYC2* $^{*}(6,178)$

STZ/ZAT10* (260)

ATAF2 $(4,317)$

NAC3/ANAC055* (377)

ERF1 $(8,042)$

ORA59 $(4,510)$

DREB2B (124)

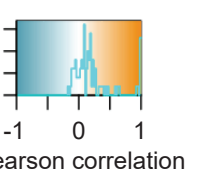


bioRxiv preprint doi: https://doi.org/10.1101/736363; this version posted August 19,2019 . The copyright holder for this preprint (which was not Fig urertifif by peer review) is the author/funder, who has granted bioRxiv a license to display the preprint in perpetuity. It is made available under

a

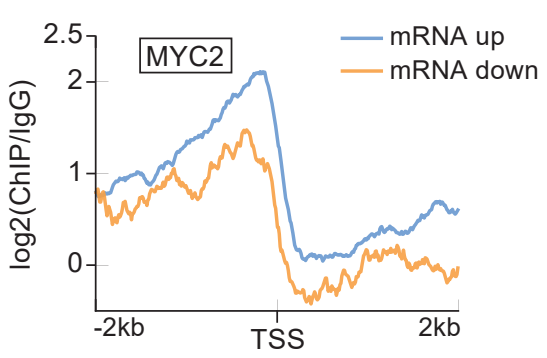

b

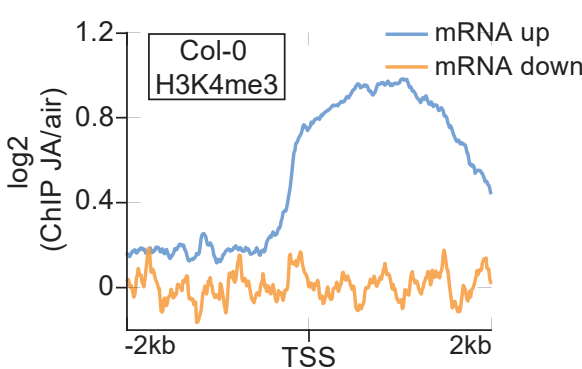

C

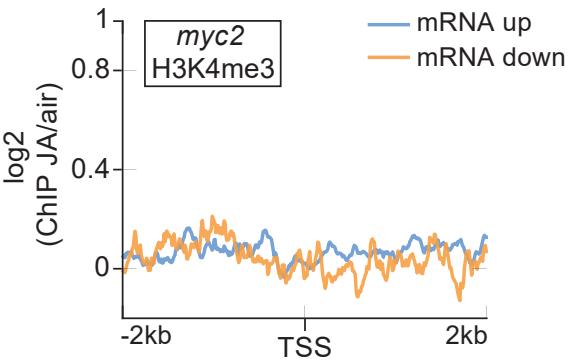

d

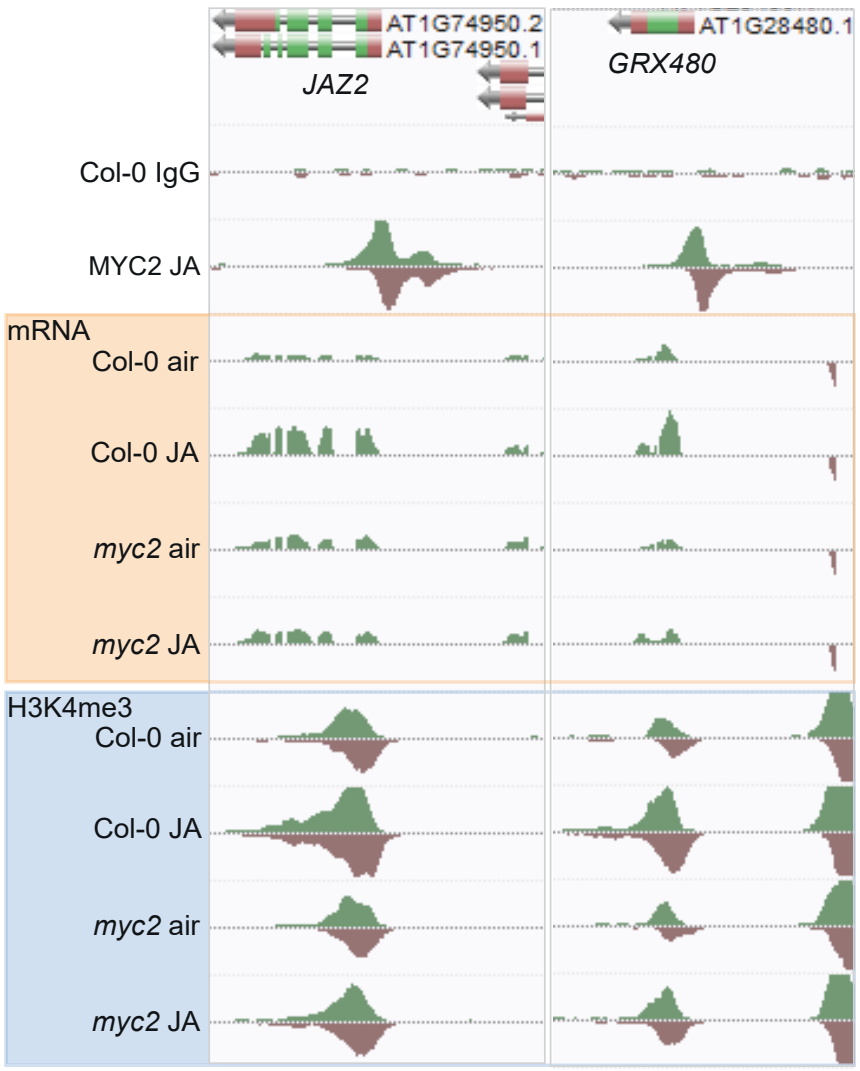


bioRxiv preprint doi: https://doi.org/10.1101/736363; this version posted August 19, 2019. The copyright holder for this preprint (which was not Fig Uqedified by peer review) is the author/funder, who has granted bioRxiv a license to display the preprint in perpetuity. It is made available under

a

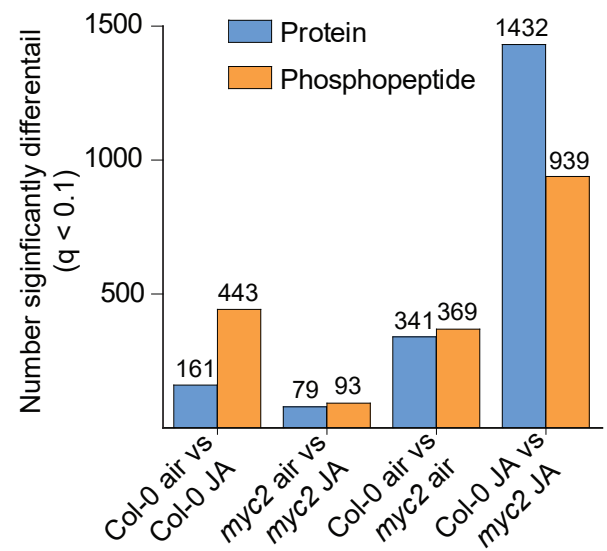

b

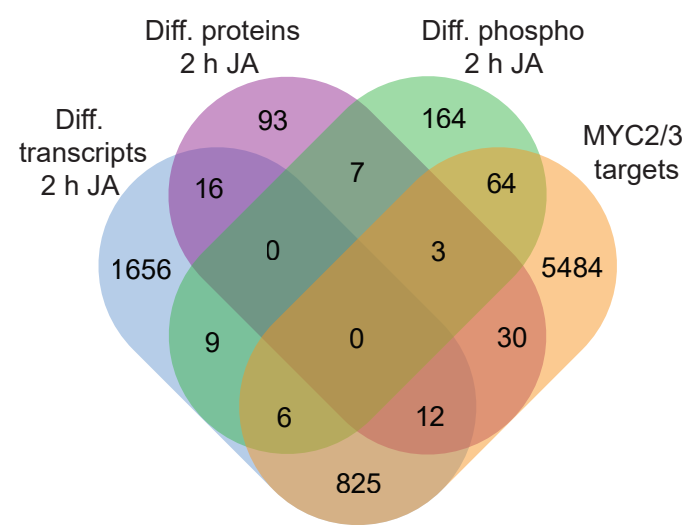

C

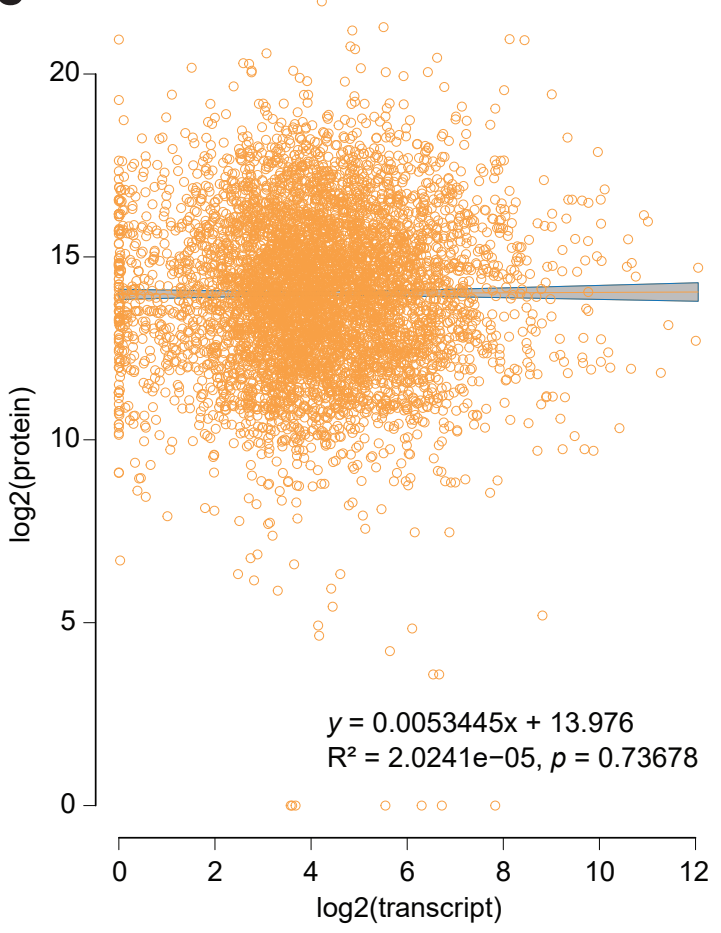

d

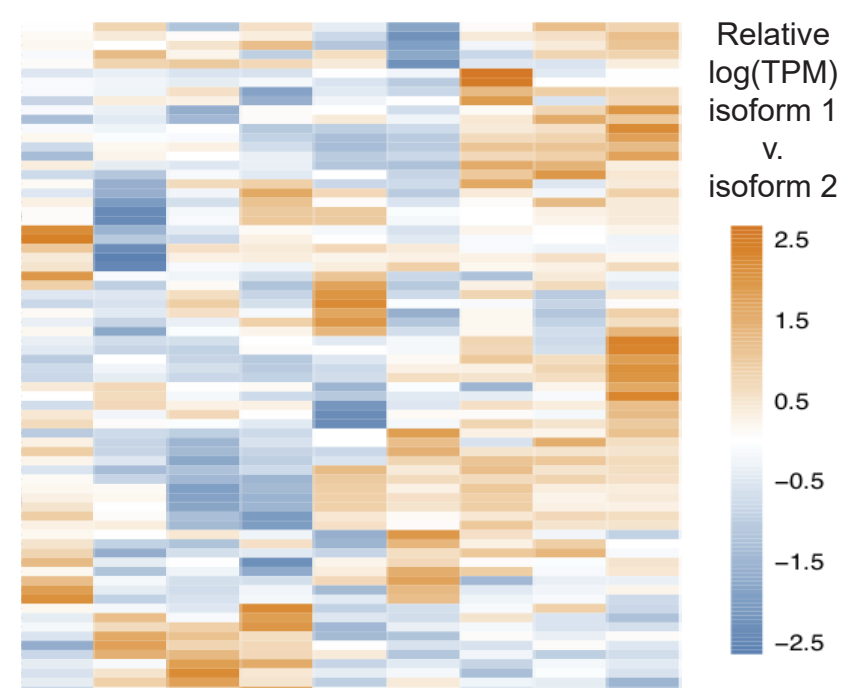

e

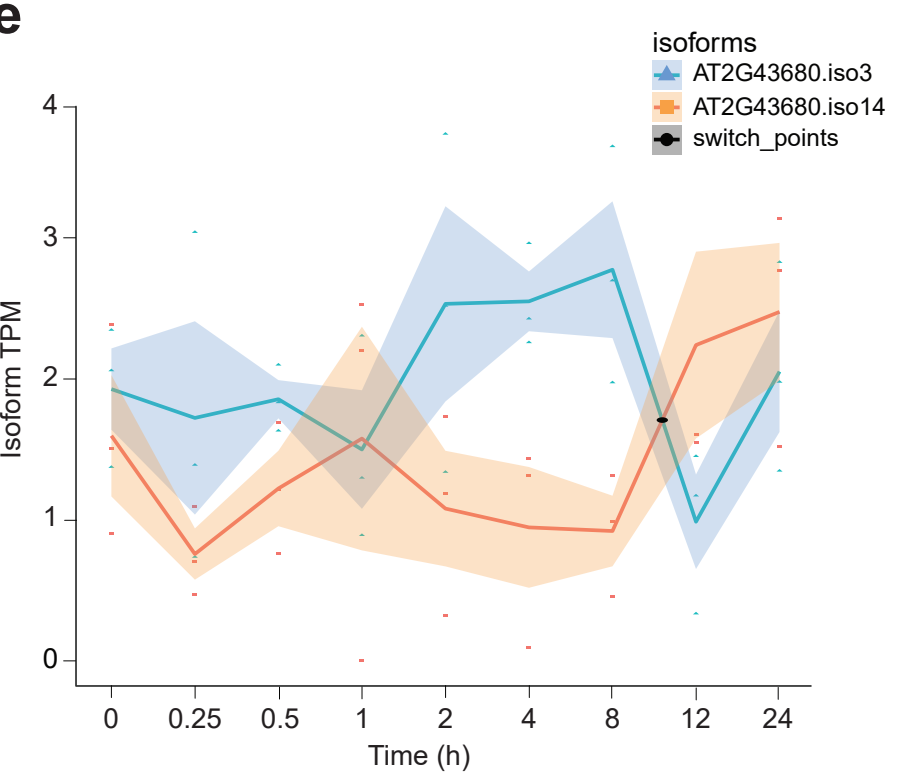


bioBxiv preprint doi: https://doi.org/10.1101/736363; this version posted August 19, 2019. The copyright holder for this preprint (which was not Fig Ur Eertfed by peer review) is the author/funder, who has granted bioRxiv a license to display the preprint in perpetuity. It is made available under

a

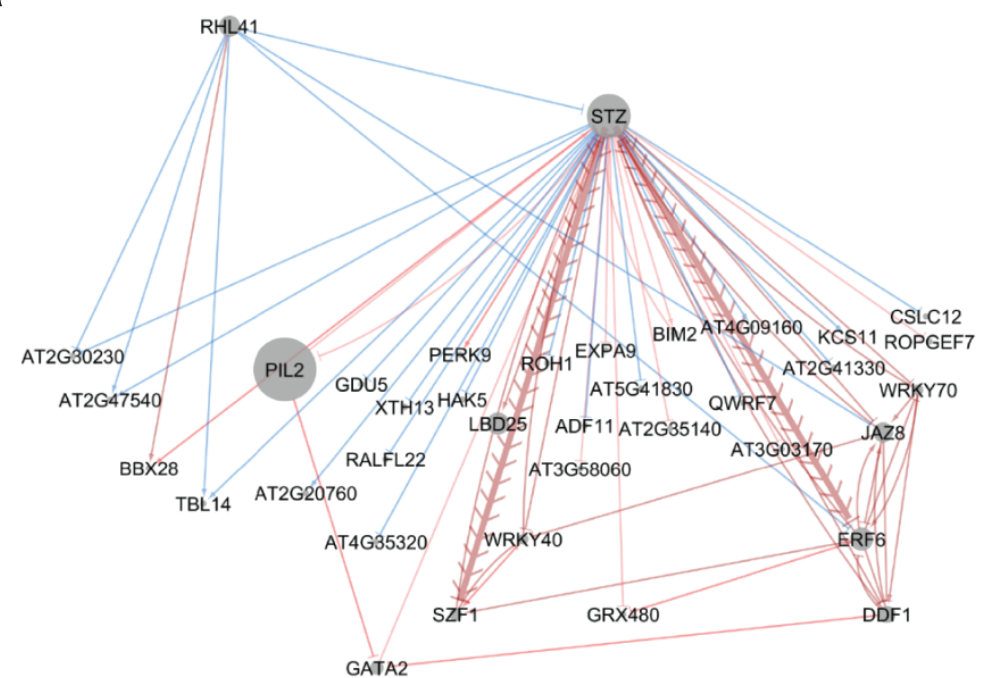

C

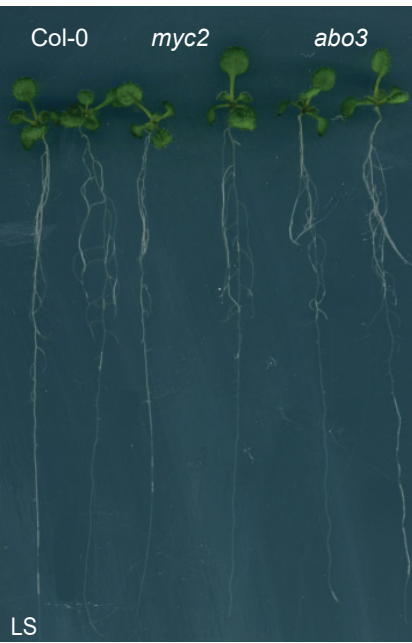

e
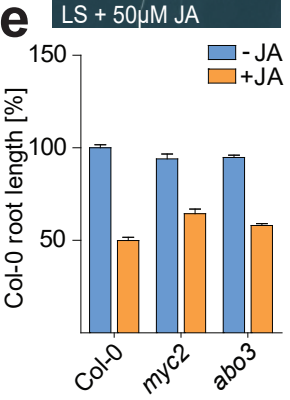

b

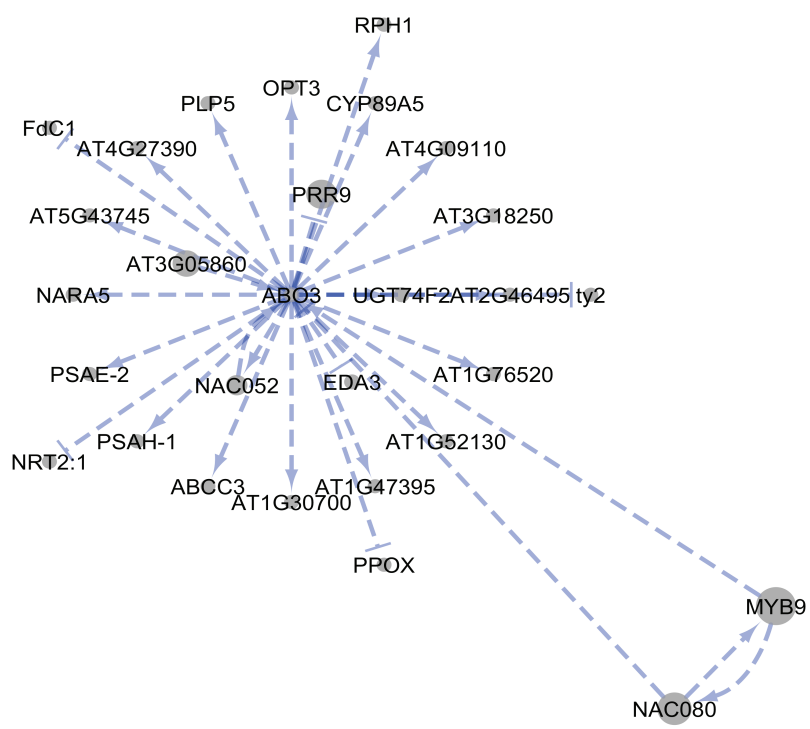

f

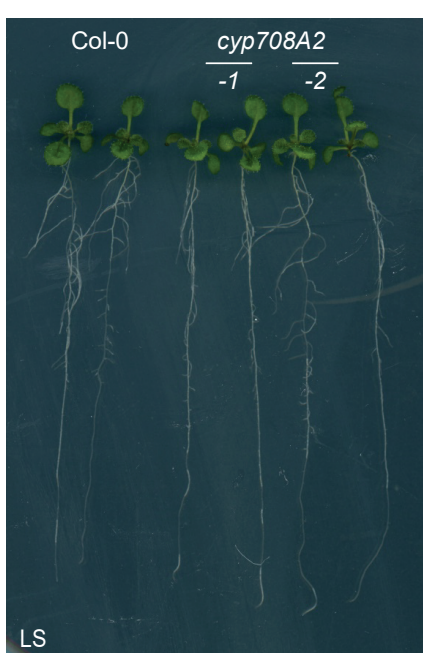

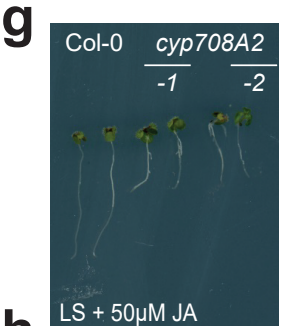

h

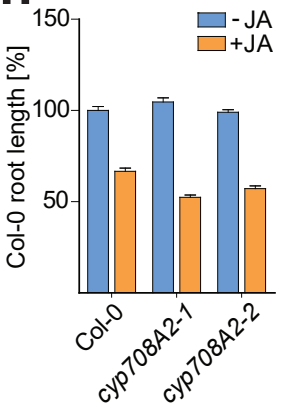

